\title{
Solving the Telegraph and Oscillatory Differential Equations by a Block Hybrid Trigonometrically Fitted Algorithm
}

\author{
F. F. Ngwane ${ }^{1}$ and S. N. Jator ${ }^{2}$ \\ ${ }^{1}$ Department of Mathematics, USC Salkehatchie, Walterboro, SC 29488, USA \\ ${ }^{2}$ Department of Mathematics and Statistics, Austin Peay State University, Clarksville, TN 37044, USA \\ Correspondence should be addressed to S. N. Jator; jators@apsu.edu
}

Received 6 July 2015; Accepted 22 October 2015

Academic Editor: Salim Messaoudi

Copyright (c) 2015 F. F. Ngwane and S. N. Jator. This is an open access article distributed under the Creative Commons Attribution License, which permits unrestricted use, distribution, and reproduction in any medium, provided the original work is properly cited.

We propose a block hybrid trigonometrically fitted (BHT) method, whose coefficients are functions of the frequency and the stepsize for directly solving general second-order initial value problems (IVPs), including systems arising from the semidiscretization of hyperbolic Partial Differential Equations (PDEs), such as the Telegraph equation. The BHT is formulated from eight discrete hybrid formulas which are provided by a continuous two-step hybrid trigonometrically fitted method with two off-grid points. The BHT is implemented in a block-by-block fashion; in this way, the method does not suffer from the disadvantages of requiring starting values and predictors which are inherent in predictor-corrector methods. The stability property of the BHT is discussed and the performance of the method is demonstrated on some numerical examples to show accuracy and efficiency advantages.

\section{Introduction}

In what follows, we consider the numerical solution of the general second order IVPs of the form

$$
\begin{aligned}
y^{\prime \prime} & =f\left(x, y, y^{\prime}\right), \\
y\left(x_{0}\right) & =y_{0}, \\
y^{\prime}\left(x_{0}\right) & =y_{0}^{\prime}, \\
& x \in\left[x_{0}, x_{N}\right],
\end{aligned}
$$

where $f: \mathbb{R} \times \mathbb{R}^{2 m} \rightarrow \mathbb{R}^{2 m}, N>0$ is an integer, and $m$ is the dimension of the system. Problems of the form of (1) frequently arise in several areas of science and engineering such as classical mechanics, celestial mechanics, quantum mechanics, control theory, circuit theory, astrophysics, and biological sciences. Equation (1) is traditionally solved by reducing it into a system of first-order IVPs of double dimension and then solved using the various methods that are available for solving systems of first-order IVPs (see
Lambert [1, 2], Hairer and Wanner in [3], Hairer [4], and Brugnano and Trigiante $[5,6])$.

Nevertheless, there are numerous methods for directly solving the special second-order IVPs in which the first derivative does not appear explicitly and it has been shown that these methods have the advantages of requiring less storage space and fewer number of function evaluations (see Hairer [4], Hairer et al. [7], Simos [8], Lambert and Watson [9], and Twizell and Khaliq [10]). Fewer methods have been proposed for directly solving second-order IVPs in which the first derivative appears explicitly (see Vigo-Aguiar and Ramos [11], Awoyemi [12], Chawla and Sharma [13], Mahmoud and Osman [14], Franco [15], and Jator [16]). It is also the case that some of these IVPs possess solutions with special properties that may be known in advance and taken advantage of when designing numerical methods. In this light, several methods have been presented in the literature which take advantage of the special properties of the solution that may be known in advance (see Coleman and Duxbury [17], Coleman and Ixaru [18], Simos [19], Vanden Berghe et al. [20], Vigo-Aguiar and Ramos [11], Fang et al. [21], Nguyen et al. [22], Ramos and 
Vigo-Aguiar [23], Franco and Gómez [24], and Ozawa [25]). However, most of these methods are restricted to solving special second-order IVPs in a predictor-corrector mode.

Our objective is to present a BHT that is implemented in a block-by-block fashion; in this way, the method does not suffer from the disadvantages of requiring starting values and predictors which are inherent in predictor-corrector methods (see Jator et al. [26], Jator [27], and Ngwane and Jator [28]). We note that multiderivative trigonometrically-fitted block methods for $y^{\prime \prime}=f\left(x, y, y^{\prime}\right)$ have been proposed in Jator [29] and Jator [27]. However, the BHT proposed in this paper avoids the computation of higher order derivatives which have the potential to increase computational cost, especially when applied to nonlinear systems. We note that Ramos et al. [30] recently proposed a trigonometrically fitted optimized two-step hybrid block method for solving the general secondorder IVPs with oscillatory solutions. However, the method given in [30] is of an order 2; hence, in this paper, we propose a BHT which is of order 5 and its application is extended to solving PDEs such as the Telegraph equation.

The organization of this paper is as follows. In Section 2, we derive the BHT for solving (1). The analysis and implementation of the BHT are discussed in Section 3. Numerical examples are given in Section 4 to show the accuracy and efficiency of the BHT. Finally, the conclusion of the paper is given in Section 5.

\section{Development of Method}

Consider

$$
\begin{aligned}
y_{n+2}= & \alpha_{2,0} y_{n}+\alpha_{2,1} y_{n+1} \\
& +h^{2} \sum_{j=0}^{2} \beta_{2, j} f_{n+j}+\beta_{2, n+v} f_{n+v}+\beta_{2, n+\epsilon} f_{n+\epsilon}, \\
y_{n+v}= & \alpha_{v, 0} y_{n}+\alpha_{v, 1} y_{n+1} \\
& +h^{2} \sum_{j=0}^{2} \beta_{v, j} f_{n+j}+\beta_{v, n+v} f_{n+v}+\beta_{v, n+\epsilon} f_{n+\epsilon}, \\
y_{n+\epsilon}= & \alpha_{\epsilon, 0} y_{n}+\alpha_{\epsilon, 1} y_{n+1} \\
& +h^{2} \sum_{j=0}^{2} \beta_{\epsilon, j} f_{n+j}+\beta_{\epsilon, n+v} f_{n+v}+\beta_{\epsilon, n+\epsilon} f_{n+\epsilon}
\end{aligned}
$$

which are used together with additional methods given as

$$
\begin{aligned}
h y_{n+2}^{\prime}= & \alpha_{2,0}^{\prime} y_{n}+\alpha_{2,1}^{\prime} y_{n+1} \\
& +h^{2} \sum_{j=0}^{2} \beta_{2, j}^{\prime} f_{n+j}+\beta_{2, n+v}^{\prime} f_{n+v}+\beta_{2, n+\epsilon}^{\prime} f_{n+\epsilon}, \\
h y_{n+v}^{\prime}= & \alpha_{v, 0}^{\prime} y_{n}+\alpha_{v, 1}^{\prime} y_{n+1} \\
& +h^{2} \sum_{j=0}^{2} \beta_{v, j}^{\prime} f_{n+j}+\beta_{v, n+v}^{\prime} f_{n+v}+\beta_{v, n+\epsilon}^{\prime} f_{n+\epsilon},
\end{aligned}
$$

$$
\begin{aligned}
h y_{n+1}^{\prime}= & \alpha_{1,0}^{\prime} y_{n}+\alpha_{1,1}^{\prime} y_{n+1} \\
& +h^{2} \sum_{j=0}^{2} \beta_{1, j}^{\prime} f_{n+j}+\beta_{1, n+v}^{\prime} f_{n+v}+\beta_{1, n+\epsilon}^{\prime} f_{n+\epsilon}, \\
h y_{n+\epsilon}^{\prime}= & \alpha_{\epsilon, 0}^{\prime} y_{n}+\alpha_{\epsilon, 1}^{\prime} y_{n+1} \\
& +h^{2} \sum_{j=0}^{2} \beta_{\epsilon, j}^{\prime} f_{n+j}+\beta_{\epsilon, n+v}^{\prime} f_{n+v}+\beta_{\epsilon, n+\epsilon}^{\prime} f_{n+\epsilon}, \\
h y_{n}^{\prime}= & \alpha_{0,0}^{\prime} y_{n}+\alpha_{0,1}^{\prime} y_{n+1} \\
& +h^{2} \sum_{j=0}^{2} \beta_{0, j}^{\prime} f_{n+j}+\beta_{0, n+v}^{\prime} f_{n+v}+\beta_{0, n+\epsilon}^{\prime} f_{n+\epsilon},
\end{aligned}
$$

where $\alpha_{j, 0}, \alpha_{j, 1}$, and $\beta_{j, j}, j=0, v, 1, \epsilon, 2$, are coefficients that depend on the step-length $h$ and frequency $w$. The coefficients of the method are chosen so that the method integrates IVP (1) exactly where the solutions are members of the linear space $\left\langle 1, x, x^{2}, x^{3}, x^{4}, \sin (w x), \cos (w x)\right\rangle$.

The main method has the form

$$
y_{n+2}=\sum_{i=0}^{1} \alpha_{i} y_{n+i}+h^{2} \sum_{j=0}^{2} \beta_{j} f_{n+j}+\beta_{n+v} f_{n+v}+\beta_{n+\epsilon} f_{n+\epsilon}
$$

where $\alpha_{i}, i=0,1$, and $\beta_{j}, j=0, v, 1, \epsilon, 2$, are to be determined coefficient functions of the frequency and stepsize. To derive the main method and additional methods, we initially seek a continuous local approximation $\Pi(x)$ on the interval $\left[x_{n}, x_{n+2}\right]$ of the form

$$
\begin{array}{r}
\Pi(x)=\alpha_{0}(x) y_{n}+\alpha_{1}(x) y_{n+1}+h^{2} \sum_{j} \beta_{j}(x) f_{n+j}, \\
\quad j=0, v, 1, \epsilon, 2
\end{array}
$$

which represents our CHT and where $\alpha_{0}(x), \alpha_{1}(x)$, and $\beta_{j}(x)$, $j=0, v, 1, \epsilon, 2$, are continuous coefficients. The first derivative of (5) is given by

$$
\Pi^{\prime}(x)=\frac{d}{d x} \Pi(x)
$$

We assume that $y_{n+j}=\Pi\left(x_{n+j}\right)$ is the numerical approximation to the analytical solution $y\left(x_{n+j}\right), y_{n+j}^{\prime}=\Pi^{\prime}\left(x_{n+j}\right)$ is the numerical approximation to $y^{\prime}\left(x_{n+j}\right)$, and $f_{n+j}=\Pi^{\prime \prime}\left(x_{n+j}\right)$ is an approximation to $y^{\prime}\left(x_{n+j}\right), j=0, v, 1, \epsilon, 2$.

The following theorem shows how continuous method (5) is constructed. This is done by requiring that on the interval from $x_{n}$ to $x_{n+2}=x_{n}+2 h$ the exact solution is locally approximated by function (5) with (6) obtained as a consequence.

Theorem 1. Let $F_{i}(x)=x^{i}, i=0,1,2,3,4, F_{5}(x)=$ $\sin w x$, and $F_{6}(x)=\cos w x$ be basis functions and $K=$ 
$\left(y_{n}, y_{n+1}, f_{n}, f_{n+v}, f_{n+1}, f_{n+\epsilon}, f_{n+2}\right)^{T}$ a vector, where $T$ is the transpose. Define the matrix $G$ by

$$
G=\left(\begin{array}{ccc}
F_{0}\left(x_{n}\right) & \cdots & F_{6}\left(x_{n}\right) \\
F_{0}\left(x_{n+1}\right) & \cdots & F_{6}\left(x_{n+1}\right) \\
F_{0}^{\prime \prime}\left(x_{n}\right) & \cdots & F_{6}^{\prime \prime}\left(x_{n}\right) \\
F_{0}^{\prime \prime}\left(x_{n+v}\right) & \cdots & F_{6}^{\prime \prime}\left(x_{n+v}\right) \\
F_{0}^{\prime \prime}\left(x_{n+1}\right) & \cdots & F_{6}^{\prime \prime}\left(x_{n+1}\right) \\
F_{0}^{\prime \prime}\left(x_{n+\epsilon}\right) & \cdots & F_{6}^{\prime \prime}\left(x_{n+\epsilon}\right) \\
F_{0}^{\prime \prime}\left(x_{n+2}\right) & \cdots & F_{6}^{\prime \prime}\left(x_{n+2}\right)
\end{array}\right)
$$

and $G_{i}$ is obtained by replacing the ith column of $G$ by the vector $K$. Let the following conditions be satisfied:

$$
\begin{aligned}
\Pi\left(x_{n}\right) & =y_{n}, \\
\Pi^{\prime}\left(x_{n}\right) & =y_{n}^{\prime}, \\
\Pi^{\prime \prime}\left(x_{n}+j\right) & =f_{n+j}, \quad j=0, v, 1, \epsilon, 2 ;
\end{aligned}
$$

then continuous representations (5) and (6) are equivalent to the following:

$$
\begin{aligned}
\Pi(x) & =\sum_{i=0}^{6} \frac{\operatorname{det}\left(G_{i}\right)}{\operatorname{det}(G)} F_{i}(x), \\
\Pi^{\prime}(x) & =\frac{d}{d x}\left(\sum_{i=0}^{6} \frac{\operatorname{det}\left(G_{i}\right)}{\operatorname{det}(G)} F_{i}(x)\right) .
\end{aligned}
$$

Proof. We use the approach given in Jator [16] with appropriate notational modification. Let method (5) be defined by the assumed basis functions:

$$
\begin{gathered}
\alpha_{0}(x)=\sum_{i=0}^{6} \alpha_{i+1,0} F_{i}(x), \\
\alpha_{1}(x)=\sum_{i=0}^{6} \alpha_{i+1,1} F_{i}(x), \\
h^{2} \beta_{j}(x)=\sum_{i=0}^{6} h^{2} \beta_{i+1, j} F_{i}(x),
\end{gathered}
$$

where $\alpha_{i+1,0}, \alpha_{i+1,1}$, and $h^{2} \beta_{i+1, j}$, are coefficients to be determined. Substituting (11) into (5), we get

$$
\begin{aligned}
\Pi(x)= & \sum_{i=0}^{6} \alpha_{i+1,0} F_{i}(x) y_{n}+\sum_{i=0}^{6} \alpha_{i+1,1} F_{i}(x) y_{n+1} \\
& +\sum_{j} \sum_{i=0}^{6} h^{2} \beta_{i+1, j} F_{i}(x) f_{n+j}
\end{aligned}
$$

which is simplified to

$$
\begin{aligned}
& \Pi(x)=\sum_{i=0}^{6}\left(\alpha_{i+1,0} F_{i}(x) y_{n}+\alpha_{i+1,1} F_{i}(x) y_{n+1}\right. \\
& \left.\quad+\sum_{j} h^{2} \beta_{i+1, j} F_{i}(x) f_{n+j}\right)
\end{aligned}
$$

and expressed as

$$
\Pi(x)=\sum_{i=0}^{6} \ell_{i} F_{i}(x),
$$

where

$$
\ell_{i}=\alpha_{i+1,0} y_{n}+\alpha_{i+1,1} y_{n+1}+\sum_{j} h^{2} \beta_{i+1, j} f_{n+j}
$$

By imposing conditions (8) on (14), we obtain a system of six equations which can be expressed as

$$
G L=K,
$$

where $L=\left(\ell_{0}, \ell_{1}, \ldots, \ell_{6}\right)^{T}$ is a vector whose coefficients are determined via Cramer's rule as

$$
\ell_{i}=\frac{\operatorname{det}\left(G_{i}\right)}{\operatorname{det}(G)}, \quad i=0,1, \ldots, 6,
$$

where $G_{i}$ is obtained by replacing the $i$ th column of $G$ by $K$. In order to obtain the continuous approximation, we use the elements of $L$ to rewrite (14) as

$$
\Pi(x)=\sum_{i=0}^{6} \frac{\operatorname{det}\left(G_{i}\right)}{\operatorname{det}(G)} F_{i}(x),
$$

whose first derivative is given by

$$
\Pi^{\prime}(x)=\frac{d}{d x}\left(\sum_{i=0}^{6} \frac{\operatorname{det}\left(G_{i}\right)}{\operatorname{det}(G)} F_{i}(x)\right) .
$$

Remark 2. In the derivation of the BHT, the basis functions $F_{i}(x)=x^{i}, i=0,1,2,3,4, F_{5}(x)=\sin w x$, and $F_{6}(x)=\cos w x$ are chosen because they are simple to analyze. Nevertheless, other possible bases are possible (see Nguyen et al. [22]).

2.1. Specification of the Method. We note that continuous methods (9) and (10) which are equivalent to forms (5) and (6) are used to generate three discrete methods and five additional methods. The discrete and additional methods are then applied as a BHT for solving (1). We choose $v=1 / 2$, $\epsilon=3 / 2$ and evaluating (9) at $x=x_{n+2}, x=x_{n+v}$, and $x=x_{n+\epsilon}$, respectively, gives the three discrete methods $y_{n+2}=$ $\Pi\left(x_{n}+2 h\right), y_{n+v}=\Pi\left(x_{n}+v h\right)$, and $y_{n+\epsilon}=\Pi\left(x_{n}+\epsilon h\right)$ which take the form of the main method. Evaluating (10) at $x=x_{n}$, 
$x=x_{n+v}, x=x_{n+1}, x=x_{n+\epsilon}$, and $x=x_{n+2}$, respectively, gives the additional methods $y_{n}^{\prime}=\Pi^{\prime}\left(x_{n}\right), y_{n+v}^{\prime}=\Pi^{\prime}\left(x_{n}+v h\right)$, $y_{n+1}^{\prime}=\Pi^{\prime}\left(x_{n}+h\right), y_{n+\epsilon}^{\prime}=\Pi^{\prime}\left(x_{n}+\epsilon h\right)$, and $y_{n+2}^{\prime}=\Pi^{\prime}\left(x_{n}+\right.$
$2 h)$. The coefficients and their corresponding Taylor series equivalence of $y_{n+v}, y_{n+\varepsilon}, y_{n+2}, h y_{n}^{\prime}, h y_{n+v}^{\prime}, h y_{n+1}^{\prime}, h y_{n+\varepsilon}^{\prime}$, and $h y_{n+2}^{\prime}$ are, respectively, given as follows:

$$
\begin{aligned}
& \alpha_{v, 0}=\frac{1}{2}, \\
& \alpha_{v, 1}=\frac{1}{2} \text {, } \\
& \beta_{v, 0}=\frac{\left(-7 u^{2}+\left(13 u^{2}-144\right) \cos (u / 2)+48 \cos (u)+96\right) \csc ^{4}(u / 4)}{768 u^{2}} \\
& =-\frac{19}{1920}-\frac{221 u^{2}}{1935360}-\frac{233 u^{4}}{232243200}-\frac{199 u^{6}}{27249868800}-\frac{1543 u^{8}}{34780741632000}-\frac{26281 u^{10}}{128549621071872000}+\cdots, \\
& \beta_{v, v}=-\frac{h^{2}\left(23 u^{2}-12\left(u^{2}+32\right) \cos (u / 2)+\left(13 u^{2}+144\right) \cos (u)+240\right) \csc ^{4}(u / 4)}{768 u^{2}} \\
& =-\frac{17 h^{2}}{160}+\frac{79 h^{2} u^{2}}{241920}+\frac{79 h^{2} u^{4}}{29030400}+\frac{61 h^{2} u^{6}}{3406233600}+\frac{4099 h^{2} u^{8}}{47823519744000}+\frac{1739 h^{2} u^{10}}{16068702633984000}+\cdots, \\
& \beta_{v, 1}=\frac{\left(\left(17 u^{2}-144\right) \cos (u / 2)+\left(u^{2}+72\right) \cos (u)+72\right) \csc ^{4}(u / 4)}{384 u^{2}} \\
& =-\frac{7}{960}-\frac{19 u^{2}}{64512}-\frac{83 u^{4}}{38707200}-\frac{u^{6}}{100925440}+\frac{577 u^{8}}{63764692992000}+\frac{773 u^{10}}{856997473812480}+\cdots, \\
& \beta_{v, \epsilon}=-\frac{\left(5 u^{2}+\left(u^{2}+48\right) \cos (u / 2)-48\right) \cot ^{2}(u / 4) \csc ^{2}(u / 4)}{192 u^{2}} \\
& =-\frac{1}{480}+\frac{u^{2}}{15120}+\frac{u^{4}}{7257600}-\frac{u^{6}}{212889600}-\frac{167 u^{8}}{1707982848000}-\frac{2633 u^{10}}{2008587829248000}+\cdots, \\
& \beta_{v, 2}=\frac{\left(5 u^{2}+\left(u^{2}+48\right) \cos (u / 2)-48\right) \csc ^{4}(u / 4)}{768 u^{2}} \\
& =\frac{1}{1920}+\frac{31 u^{2}}{1935360}+\frac{67 u^{4}}{232243200}+\frac{109 u^{6}}{27249868800}+\frac{18127 u^{8}}{382588157952000}+\frac{64931 u^{10}}{128549621071872000}+\cdots, \\
& \alpha_{\epsilon, 0}=-\frac{1}{2} \\
& \alpha_{\epsilon, 1}=\frac{3}{2} \text {, } \\
& \beta_{\epsilon, 0}=-\frac{\left(u^{2}+\left(5 u^{2}-16\right) \cos (u / 2)+16 \cos (u)\right) \csc ^{4}(u / 4)}{256 u^{2}} \\
& =\frac{17}{1920}+\frac{53 u^{2}}{645120}+\frac{11 u^{4}}{25804800}-\frac{19 u^{6}}{27249868800}-\frac{6427 u^{8}}{127529385984000}-\frac{11509 u^{10}}{14283291230208000}+\cdots, \\
& \beta_{\epsilon, v}=\frac{\left(23 u^{2}-4 u^{2} \cos (u / 2)+\left(5 u^{2}+48\right) \cos (u)-48\right) \csc ^{4}(u / 4)}{256 u^{2}} \\
& =\frac{21}{160}-\frac{u^{2}}{5040}-\frac{u^{4}}{2419200}+\frac{u^{6}}{70963200}+\frac{167 u^{8}}{569327616000}+\frac{2633 u^{10}}{669529276416000}+\cdots, \\
& \beta_{\epsilon, 1}=\frac{3\left(-\left(7 u^{2}+16\right) \cos (u / 2)+\left(u^{2}-8\right) \cos (u)+24\right) \csc ^{4}(u / 4)}{128 u^{2}} \\
& =\frac{67}{320}+\frac{11 u^{2}}{107520}-\frac{17 u^{4}}{12902400}-\frac{173 u^{6}}{4541644800}-\frac{12277 u^{8}}{21254897664000}-\frac{49729 u^{10}}{7141645615104000}+\cdots,
\end{aligned}
$$




$$
\begin{aligned}
& \beta_{\epsilon, \epsilon}=\frac{\left(19 u^{2}+4\left(u^{2}+32\right) \cos (u / 2)+\left(u^{2}+16\right) \cos (u)-144\right) \csc ^{4}(u / 4)}{256 u^{2}} \\
& =\frac{13}{480}+\frac{u^{2}}{16128}+\frac{u^{4}}{460800}+\frac{5 u^{6}}{136249344}+\frac{691 u^{8}}{1449197568000}+\frac{u^{10}}{186856243200}+\cdots, \\
& \beta_{\epsilon, 2}=-\frac{\left(5 u^{2}+\left(u^{2}+48\right) \cos (u / 2)-48\right) \csc ^{4}(u / 4)}{256 u^{2}} \\
& =-\frac{1}{640}-\frac{31 u^{2}}{645120}-\frac{67 u^{4}}{77414400}-\frac{109 u^{6}}{9083289600}-\frac{18127 u^{8}}{127529385984000}-\frac{64931 u^{10}}{42849873690624000}+\cdots, \\
& \alpha_{2,0}=1, \\
& \alpha_{2,1}=2 \text {, } \\
& \beta_{2,0}=-\frac{\left(u^{2}+2 u^{2} \cos (u / 2)+6 \cos (u)-6\right) \csc ^{4}(u / 4)}{48 u^{2}} \\
& =\frac{1}{60}+\frac{u^{2}}{7560}+\frac{u^{4}}{3628800}-\frac{u^{6}}{106444800}-\frac{167 u^{8}}{853991424000}-\frac{2633 u^{10}}{1004293914624000}+\cdots, \\
& \beta_{2, v}=\frac{\left(5 u^{2}+\left(u^{2}+12\right) \cos (u)-12\right) \csc ^{4}(u / 4)}{24 u^{2}}=\frac{4}{15}-\frac{u^{2}}{1890}-\frac{u^{4}}{907200}+\frac{u^{6}}{26611200}+\frac{167 u^{8}}{213497856000}+\frac{2633 u^{10}}{251073478656000}+\cdots, \\
& \beta_{2,1}=\frac{\left(-10 u^{2} \cos (u / 2)+\left(u^{2}-18\right) \cos (u)+18\right) \csc ^{4}(u / 4)}{24 u^{2}} \\
& =\frac{13}{30}+\frac{u^{2}}{1260}+\frac{u^{4}}{604800}-\frac{u^{6}}{17740800}-\frac{167 u^{8}}{142331904000}-\frac{2633 u^{10}}{167382319104000}+\cdots, \\
& \beta_{2, \epsilon}=\frac{\left(5 u^{2}+\left(u^{2}+12\right) \cos (u)-12\right) \csc ^{4}(u / 4)}{24 u^{2}}=\frac{4}{15}-\frac{u^{2}}{1890}-\frac{u^{4}}{907200}+\frac{u^{6}}{26611200}+\frac{167 u^{8}}{213497856000}+\frac{2633 u^{10}}{251073478656000}+\cdots, \\
& \beta_{2,2}=-\frac{\left(u^{2}+2 u^{2} \cos (u / 2)+6 \cos (u)-6\right) \csc ^{4}(u / 4)}{48 u^{2}} \\
& =\frac{1}{60}+\frac{u^{2}}{7560}+\frac{u^{4}}{3628800}-\frac{u^{6}}{106444800}-\frac{167 u^{8}}{853991424000}-\frac{2633 u^{10}}{1004293914624000}+\cdots, \\
& \alpha_{0,0}^{\prime}=-1 \text {, } \\
& \alpha_{0,1}^{\prime}=1 \text {, } \\
& \beta_{0,0}^{\prime}=\frac{\csc ^{4}(u / 4)\left(-7 u^{2}+10 u^{2} \cos (u / 2)-6 \cos (5 u / 4) \sec (u / 4)-6 u \sin (5 u / 4) \sec (u / 4)+6\right)}{96 u^{2}} \\
& =-\frac{53}{360}-\frac{19 u^{2}}{15120}-\frac{37 u^{4}}{2419200}-\frac{163 u^{6}}{638668800}-\frac{461287 u^{8}}{83691159552000}-\frac{12773 u^{10}}{95647039488000}+\cdots, \\
& \beta_{0, v}^{\prime} \\
& =-\frac{\csc ^{4}(u / 4) \sec (u / 4)\left(5 u^{2} \cos (5 u / 4)+6\left(u^{2}+4\right) \cos (u / 4)+\left(u^{2}-6\right) \cos (3 u / 4)-6 u \sin (3 u / 4)-18 u \sin (5 u / 4)-18 \cos (5 u / 4)\right)}{96 u^{2}} \\
& =-\frac{2}{5}+\frac{13 u^{2}}{3780}+\frac{29 u^{6}}{53222400}+\frac{67 u^{4}}{1814400}+\frac{46559 u^{8}}{4184557977600}+\frac{26753 u^{10}}{100429391462400}+\cdots, \\
& \beta_{0,1}^{\prime}=\frac{\csc ^{4}(u / 4)\left(2 u^{2} \cos (u / 2)+\left(u^{2}-6\right) \cos (u)-6 u \sin (u)+6\right)}{16 u^{2}} \\
& =\frac{1}{12}-\frac{u^{2}}{360}-\frac{23 u^{4}}{1209600}-\frac{331 u^{8}}{1072963584000}-\frac{u^{6}}{9676800}+\frac{703 u^{10}}{334764638208000}+\cdots,
\end{aligned}
$$




$$
\begin{aligned}
& \beta_{0, \epsilon}^{\prime} \\
& =-\frac{\csc ^{4}(u / 4) \sec (u / 4)\left(u^{2} \cos (5 u / 4)+6\left(u^{2}+4\right) \cos (u / 4)+\left(5 u^{2}-18\right) \cos (3 u / 4)-18 u \sin (3 u / 4)-6 u \sin (5 u / 4)-6 \cos (5 u / 4)\right)}{96 u^{2}} \\
& =-\frac{2}{45}-\frac{u^{4}}{86400}+\frac{u^{2}}{3780}-\frac{13 u^{6}}{31933440}-\frac{32027 u^{8}}{2988969984000}-\frac{15019 u^{10}}{55794106368000}+\cdots, \\
& \beta_{0,2}^{\prime}=\frac{\csc ^{4}(u / 4)\left(u^{2}+2\left(u^{2}-6\right) \cos (u / 2)-6 u \sin (3 u / 4) \sec (u / 4)+12\right)}{96 u^{2}} \\
& =\frac{1}{120}+\frac{u^{2}}{3024}+\frac{13 u^{4}}{1451520}+\frac{47 u^{6}}{212889600}+\frac{452681 u^{8}}{83691159552000}+\frac{269639 u^{10}}{2008587829248000}+\cdots, \\
& \alpha_{v, 0}^{\prime}=-1 \\
& \alpha_{v, 1}^{\prime}=1 \\
& \beta_{v, 0}^{\prime}=\frac{\csc ^{3}(u / 4) \sec (u / 4)\left(\left(u^{2}+8\right) \sin (u / 2)-4 u+8 \sin (u)-8 u \cos (u / 2)\right)}{64 u^{2}} \\
& =\frac{13}{480}+\frac{17 u^{2}}{32256}+\frac{67 u^{4}}{6451200}+\frac{1549 u^{6}}{6812467200}+\frac{1205803 u^{8}}{223176425472000}+\frac{9739 u^{10}}{72873934848000}+\cdots, \\
& \beta_{v, v}^{\prime}=-\frac{\left(\left(u^{2}+24\right) \sin (u / 2)-12 u\right)(3 \cos (u / 2)+2) \csc ^{3}(u / 4) \sec (u / 4)}{96 u^{2}} \\
& =\frac{7}{144}-\frac{109 u^{2}}{80640}-\frac{139 u^{4}}{5806080}-\frac{4951 u^{6}}{10218700800}-\frac{246931 u^{8}}{22317642547200}-\frac{4329211 u^{10}}{16068702633984000}+\cdots, \\
& \beta_{v, 1}^{\prime}=\frac{\csc ^{4}(u / 4)\left(u^{2} \cos (u / 2)-\left(u^{2}+18\right) \cos (u)-18 u \sin (u / 2)+18\right)}{48 u^{2}} \\
& =-\frac{1}{10}+\frac{u^{2}}{1120}+\frac{23 u^{4}}{2419200}+\frac{19 u^{6}}{212889600}+\frac{7213 u^{8}}{9299017728000}+\frac{4289 u^{10}}{669529276416000}+\cdots, \\
& \beta_{v, \epsilon}^{\prime}=-\frac{\left(\left(u^{2}+24\right) \sin (u / 2)-12 u\right)(\cos (u / 2)+2) \csc ^{3}(u / 4) \sec (u / 4)}{96 u^{2}} \\
& =\frac{7}{240}+\frac{13 u^{2}}{80640}+\frac{109 u^{4}}{9676800}+\frac{83 u^{6}}{227082240}+\frac{1119247 u^{8}}{111588212736000}+\frac{1397321 u^{10}}{5356234211328000}+\cdots, \\
& \beta_{v, 2}^{\prime}=\frac{\csc ^{2}(u / 4)\left(u^{2}-12 u \csc (u / 2)+24\right)}{96 u^{2}} \\
& =-\frac{7}{1440}-\frac{37 u^{2}}{161280}-\frac{419 u^{4}}{58060800}-\frac{577 u^{6}}{2919628800}-\frac{577 u^{6}}{2919628800}-\frac{1148099 u^{8}}{223176425472000}-\frac{169051 u^{10}}{1285496210718720}+\cdots, \\
& \alpha_{1,0}^{\prime}=-1 \text {, } \\
& \alpha_{1,1}^{\prime}=1 \\
& \beta_{1,0}^{\prime}=-\frac{\csc ^{4}(u / 4) \sec (u / 4)\left(2 u^{2} \cos (3 u / 4)+\left(u^{2}-6\right) \cos (u / 4)+6(u \sin (u / 4)+\cos (5 u / 4))\right)}{96 u^{2}} \\
& =\frac{1}{72}-\frac{u^{2}}{30240}-\frac{u^{4}}{172800}-\frac{61 u^{6}}{319334400}-\frac{215521 u^{8}}{41845579776000}-\frac{1769 u^{10}}{13390585528320}+\cdots, \\
& \beta_{1, v}^{\prime}=\frac{\csc ^{4}(u / 4) \sec (u / 4)\left(u^{2} \cos (5 u / 4)+\left(5 u^{2}-12\right) \cos (u / 4)+6 u \sin (u / 4)+3 \cos (3 u / 4)+9 \cos (5 u / 4)\right)}{48 u^{2}} \\
& =\frac{13}{45}-\frac{u^{2}}{15120}+\frac{41 u^{4}}{3628800}+\frac{5 u^{6}}{12773376}+\frac{17569 u^{8}}{1673823191040}+\frac{267983 u^{10}}{1004293914624000}+\cdots,
\end{aligned}
$$




$$
\begin{aligned}
& \beta_{1,1}^{\prime}=\frac{\left(-10 u^{2} \cos (u / 2)+\left(u^{2}-18\right) \cos (u)+18\right) \csc ^{4}(u / 4)}{48 u^{2}} \\
& =\frac{13}{60}+\frac{u^{2}}{2520}+\frac{u^{4}}{1209600}-\frac{u^{6}}{35481600}-\frac{167 u^{8}}{284663808000}-\frac{2633 u^{10}}{334764638208000}+\cdots, \\
& \beta_{1, \epsilon}^{\prime}=\frac{\csc ^{4}(u / 4) \sec (u / 4)\left(\left(5 u^{2}-12\right) \cos (u / 4)+\left(u^{2}+9\right) \cos (3 u / 4)+3(\cos (5 u / 4)-2 u \sin (u / 4))\right)}{48 u^{2}} \\
& =-\frac{1}{45}-\frac{u^{2}}{2160}-\frac{u^{4}}{80640}-\frac{113 u^{6}}{319334400}-\frac{406493 u^{8}}{41845579776000}-\frac{85817 u^{10}}{334764638208000}+\cdots, \\
& \beta_{1,2}^{\prime}=-\frac{\csc ^{4}(u / 4) \sec (u / 4)\left(\left(u^{2}-2\right) \cos (u / 4)+2(\cos (3 u / 4)-u \sin (u / 4))\right)}{32 u^{2}} \\
& =\frac{1}{360}+\frac{u^{2}}{6048}+\frac{11 u^{4}}{1814400}+\frac{29 u^{6}}{159667200}+\frac{103669 u^{8}}{20922789888000}+\frac{5911 u^{10}}{45649723392000}+\cdots, \\
& \alpha_{\epsilon, 0}^{\prime}=-1, \\
& \alpha_{\epsilon, 1}^{\prime}=1 \\
& \beta_{\epsilon, 0}^{\prime}=-\frac{\csc ^{4}(u / 4) \sec (u / 4)\left(7 u^{2} \cos (3 u / 4)+\left(17 u^{2}-24\right) \cos (u / 4)+24(\cos (5 u / 4)-u \sin (u / 4))\right)}{384 u^{2}} \\
& =\frac{31}{1440}+\frac{5 u^{2}}{13824}+\frac{29 u^{4}}{3870720}+\frac{3847 u^{6}}{20437401600}+\frac{3313369 u^{8}}{669529276416000}+\frac{28177 u^{10}}{218621804544000}+\cdots, \\
& \beta_{\epsilon, v}^{\prime} \\
& =\frac{\csc ^{4}(u / 4) \sec (u / 4)\left(7 u^{2} \cos (5 u / 4)+12\left(7 u^{2}-8\right) \cos (u / 4)+\left(5 u^{2}+24\right) \cos (3 u / 4)-72 u \sin (u / 4)-24 u \sin (3 u / 4)+72 \cos (5 u / 4)\right)}{384 u^{2}} \\
& =\frac{19}{80}-\frac{167 u^{2}}{241920}-\frac{359 u^{4}}{29030400}-\frac{1117 u^{6}}{3406233600}-\frac{47629 u^{8}}{5150225203200}-\frac{4023451 u^{10}}{16068702633984000}+\cdots, \\
& \beta_{\epsilon, 1}^{\prime}=\frac{\csc ^{4}(u / 4)\left(-7 u^{2} \cos (u / 2)+\left(u^{2}-6\right) \cos (u)+6 u \sin (u / 2)+6\right)}{16 u^{2}} \\
& =\frac{8}{15}-\frac{u^{2}}{10080}-\frac{19 u^{4}}{2419200}-\frac{31 u^{6}}{212889600}-\frac{54371 u^{8}}{27897053184000}-\frac{14821 u^{10}}{669529276416000}+\cdots, \\
& \beta_{\epsilon, \epsilon}^{\prime} \\
& =\frac{\csc ^{4}(u / 4) \sec (u / 4)\left(5 u^{2} \cos (5 u / 4)+12\left(7 u^{2}-8\right) \cos (u / 4)+\left(7 u^{2}+72\right) \cos (3 u / 4)-24 u \sin (u / 4)-72 u \sin (3 u / 4)+24 \cos (5 u / 4)\right)}{384 u^{2}} \\
& =\frac{157}{720}+\frac{199 u^{2}}{241920}+\frac{221 u^{4}}{9676800}+\frac{97 u^{6}}{185794560}+\frac{3965821 u^{8}}{334764638208000}+\frac{499747 u^{10}}{1785411403776000}+\cdots, \\
& \beta_{\epsilon, 2}^{\prime}=-\frac{\csc ^{4}(u / 4)\left(7 u^{2}+\left(5 u^{2}+24\right) \cos (u / 2)-12 u \sin (3 u / 4) \sec (u / 4)-24\right)}{192 u^{2}} \\
& =-\frac{1}{96}-\frac{191 u^{2}}{483840}-\frac{587 u^{4}}{58060800}-\frac{1613 u^{6}}{6812467200}-\frac{3748337 u^{8}}{669529276416000}-\frac{79621 u^{10}}{584316459417600}+\cdots, \\
& \alpha_{2,0}^{\prime}=-1 \text {, } \\
& \alpha_{2,1}^{\prime}=1 \text {, } \\
& \beta_{2,0}^{\prime}=-\frac{(2 \cos (u / 2)+1) \csc ^{4}(u / 4) \sec (u / 4)\left(\left(u^{2}-2\right) \cos (u / 4)+2(\cos (3 u / 4)-u \sin (u / 4))\right)}{32 u^{2}} \\
& =\frac{1}{120}-\frac{u^{2}}{5040}-\frac{u^{4}}{115200}-\frac{7 u^{6}}{30412800}-\frac{156349 u^{8}}{27897053184000}-\frac{6109 u^{10}}{44635285094400}+\cdots,
\end{aligned}
$$




$$
\begin{aligned}
& \beta_{2, v}^{\prime}=\frac{\csc ^{4}(u / 4) \sec (u / 4)\left(\left(26 u^{2}-24\right) \cos (u / 4)+\left(7 u^{2}+6\right) \cos (3 u / 4)+3\left(\left(u^{2}+6\right) \cos (5 u / 4)-2 u(3 \sin (3 u / 4)+\sin (5 u / 4))\right)\right)}{96 u^{2}} \\
& =\frac{14}{45}-\frac{u^{2}}{1260}+\frac{19 u^{4}}{1814400}+\frac{71 u^{6}}{159667200}+\frac{2291 u^{8}}{199264665600}+\frac{12767 u^{10}}{45649723392000}+\cdots, \\
& \beta_{2,1}^{\prime}=-\frac{\csc ^{4}(u / 4)\left(26 u^{2} \cos (u / 2)+\left(u^{2}+18\right) \cos (u)-18(u \sin (u)+1)\right)}{48 u^{2}} \\
& =\frac{7}{20}+\frac{u^{2}}{280}+\frac{u^{4}}{48384}+\frac{u^{6}}{21288960}-\frac{4021 u^{8}}{4649508864000}-\frac{5969 u^{10}}{334764638208000}+\cdots, \\
& \beta_{2, \epsilon}^{\prime} \\
& =\frac{\csc ^{4}(u / 4) \sec (u / 4)\left(7 u^{2} \cos (5 u / 4)+\left(26 u^{2}-24\right) \cos (u / 4)+3\left(u^{2}+6\right) \cos (3 u / 4)-6 u \sin (3 u / 4)-18 u \sin (5 u / 4)+6 \cos (5 u / 4)\right)}{96 u^{2}} \\
& =\frac{2}{3}-\frac{u^{2}}{252}-\frac{23 u^{4}}{604800}-\frac{u^{6}}{1971200}-\frac{72143 u^{8}}{6974263296000}-\frac{6119 u^{10}}{23911759872000}+\cdots, \\
& \beta_{2,2}^{\prime}=-\frac{\csc ^{4}(u / 4)\left(-5 u^{2}+2\left(7 u^{2}+6\right) \cos (u / 2)-6 u \sin (5 u / 4) \sec (u / 4)-12\right)}{96 u^{2}} \\
& =\frac{59}{360}+\frac{u^{2}}{720}+\frac{113 u^{4}}{7257600}+\frac{157 u^{6}}{638668800}+\frac{148307 u^{8}}{27897053184000}+\frac{262967 u^{10}}{2008587829248000}+\cdots
\end{aligned}
$$

Remark 3. We note that the Taylor series expansions in (20) through (27) must be used when $u \rightarrow 0$ because the corresponding trigonometric coefficients given in these equations are vulnerable to heavy cancelations (see [8]).

2.2. Block Form. In this subsection, the BHT method is formulated from the eight discrete hybrid formulas stated in (2) and (3). We emphasize that these eight formulas are provided by the continuous two-step hybrid trigonometrically fitted method with two off-grid points given by (5) and its first derivative (6). First, we define the following vectors:

$$
\begin{aligned}
& Y_{\mu+1} \\
& \quad=\left[y_{n+v}, y_{n+1}, y_{n+\epsilon}, y_{n+2}, h y_{n+v}^{\prime}, h y_{n+1}^{\prime}, h y_{n+\epsilon}^{\prime}, h y_{n+2}^{\prime}\right]^{T}, \\
& Y_{\mu}=\left[y_{n-\epsilon}, y_{n-1}, y_{n-v}, y_{n}, h y_{n-v}^{\prime}, h y_{n-1}^{\prime}, h y_{n-\epsilon}^{\prime}, h y_{n}^{\prime}\right]^{T}, \\
& F_{\mu+1} \\
& =\left[f_{n+v}, f_{n+1}, f_{n+\epsilon}, f_{n+2}, h f_{n+v}^{\prime}, h f_{n+1}^{\prime}, h f_{n+\epsilon}, h f_{n+2}^{\prime}\right]^{T}, \\
& F_{\mu}=\left[f_{n-\epsilon}, f_{n-1}, f_{n-v}, f_{n}, h f_{n-v}^{\prime}, h f_{n-1}^{\prime}, h f_{n-\epsilon}^{\prime}, h f_{n}^{\prime}\right]^{T},
\end{aligned}
$$

where $\mu=0, \ldots, N ; n=0, \ldots, N$. The three discrete methods whose coefficients are specified by (2) and the five additional methods in (3) whose coefficients are specified by (20) to (27) are combined to give the BHT method, which is expressed as

$$
A_{1} Y_{\mu+1}=A_{0} Y_{\mu}+h^{2}\left(B_{0} F_{\mu}+B_{1} F_{\mu+1}\right) \text {, }
$$

where $A_{0}, A_{1}, B_{0}$, and $B_{1}$ are matrices of dimension eight whose elements characterize the method and are given by the coefficients of (2) and (3).

\section{Error Analysis and Stability}

3.1. Local Truncation Error (LTE). Define the local truncation error of (29) as

$$
L[Z(x) ; h]=Z_{\mu+1}-\left[A Z_{\mu}+h^{2} B \bar{F}_{\mu}+h^{2} C \bar{F}_{\mu+1}\right]
$$

where

$$
\begin{gathered}
Z_{\mu+1}=\left[y\left(x_{n+v}\right), y\left(x_{n+1}\right), y\left(x_{n+\epsilon}\right), y\left(x_{n+2}\right),\right. \\
\left.h y^{\prime}\left(x_{n+v}\right), h y^{\prime}\left(x_{n+1}\right), h y^{\prime}\left(x_{n+\epsilon}\right), h y^{\prime}\left(x_{n+2}\right)\right]^{T}, \\
Z_{\mu}=\left[y\left(x_{n-\epsilon}\right), y\left(x_{n-1}\right), y\left(x_{n-v}\right), y\left(x_{n}\right), h y^{\prime}\left(x_{n-\epsilon}\right),\right. \\
\left.h y^{\prime}\left(x_{n-1}\right), h y^{\prime}\left(x_{n-v}\right), h y^{\prime}\left(x_{n}\right)\right]^{T}, \\
\bar{F}_{\mu+1}=\left[f\left(x_{n+\epsilon}, y_{n+\epsilon}\right), f\left(x_{n+1}, y_{n+1}\right), f\left(x_{n+v}, y_{n+v}\right),\right. \\
f\left(x_{n+2}, y_{n+2}\right), h f^{\prime}\left(x_{n+v}, y_{n+v}\right), h f^{\prime}\left(x_{n+1}, y_{n+1}\right), \\
\left.h f^{\prime}\left(x_{n+\epsilon}, y_{n+\epsilon}\right), h f^{\prime}\left(x_{n+2}, y_{n+2}\right)\right]^{T}, \\
\bar{F}_{\mu}=\left[f\left(x_{n-\epsilon}, y_{n-\epsilon}\right), f\left(x_{n-1}, y_{n-1}\right), f\left(x_{n-v}, y_{n-v}\right),\right. \\
f\left(x_{n}, y_{n}\right), h f^{\prime}\left(x_{n-\epsilon}, y_{n-\epsilon}\right), h f^{\prime}\left(x_{n-1}, y_{n-1}\right), \\
\left.h f^{\prime}\left(x_{n-v}, y_{n-v}\right), h f^{\prime}\left(x_{n}, y_{n}\right)\right]^{T}, \\
L[Z(x) ; h]=\left[L_{1}[z(x) ; h], L_{2}[z(x) ; h], \ldots,\right. \\
\left.L L_{8}[z(x) ; h]\right]^{T} \text { is linear different operator. }
\end{gathered}
$$


Suppose that $Z(x)$ is sufficiently differentiable. Then, a Taylor series expansion of the terms in (30) about the point $x$ gives the following expression for local truncation error:

$$
\begin{aligned}
L[Z(x) ; h]= & C_{0} Z(x)+C_{1} h Z^{\prime}(x)+\cdots \\
& +C_{q} h^{q} Z^{q}(x)+\cdots,
\end{aligned}
$$

where $C_{i}, i=0,1, \ldots$, are constant coefficients (see [16]).

Definition 4. Block method (29) has algebraic order of at least $p \geq 1$ provided there exists a constant $C_{p+2} \neq 0$ such that the local truncation error $E_{\mu}$ satisfies $\left\|E_{\mu}\right\|=C_{p+2} h^{p+2}+O\left(h^{p+3}\right)$, where $\|\cdot\|$ is the maximum norm.

Remark 5. (i) The local truncation error constants $\left(\bar{C}_{p+2}\right)$ of $\quad\left(y_{n+v}, y_{n+1}, y_{n+\epsilon}, y_{n+2}, h y_{n+v}^{\prime}, h y_{n+1}^{\prime}, h y_{n+\epsilon}^{\prime}, h y_{n+2}^{\prime}\right)^{T} \quad$ of block method (29) are given, respectively, by $\bar{C}_{7}=$ $(-1 / 61440,-1 / 5040,1 / 61440,0,-1 / 5040, \quad 61 / 645120$, $-1 / 40320,61 / 645120,-1 / 5040)^{T}$, where $\bar{C}_{0}=\bar{C}_{1}=\bar{C}_{2}=$ $\bar{C}_{3}=\bar{C}_{4}=\bar{C}_{5}=\bar{C}_{6}=0$.

(ii) From the local truncation error constant computation, it follows that method (29) has order $p$ of at least five.

3.2. Stability. The linear stability of the BHT is discussed by applying the method to the test equation $y^{\prime \prime}=-\lambda^{2} y$, where $\lambda$ is a real constant (see [17]). Letting $\Upsilon=\lambda h$, it is easily shown as in [18] that the application of (29) to the test equation yields

$$
\begin{aligned}
& Y_{\mu+1}=M\left(\Upsilon^{2} ; u\right) Y_{\mu}, \\
& M\left(\Upsilon^{2} ; u\right):=\left(A_{1}-\Upsilon^{2} B_{1}\right)^{-1}\left(A_{0}+\Upsilon^{2} B_{0}\right),
\end{aligned}
$$

where the matrix $M\left(\Upsilon^{2} ; u\right)$ is the amplification matrix which determines the stability of the method. In the spirit of [21], the spectral radius of $\rho\left(M\left(\Upsilon^{2} ; u\right)\right)$ can be obtained from the characteristics equation

$$
\rho^{2}-2 \Gamma\left(\Upsilon^{2} ; u\right) \rho+\Theta\left(\Upsilon^{2} ; u\right)=0,
$$

where $\Gamma\left(\Upsilon^{2} ; u\right)=\operatorname{trace} M\left(\Upsilon^{2} ; u\right)$ and $\Theta\left(\Upsilon^{2} ; u\right)=$ $\operatorname{det} M\left(\Upsilon^{2} ; u\right)$ are rational functions.

Definition 6. A region of stability is a region in the $q-u$ plane, throughout which $\rho\left(M\left(\Upsilon^{2} ; u\right)\right) \leq 1$ and any closed curve given by $\rho\left(M\left(\Upsilon^{2} ; u\right)\right)=1$ defines the stability boundary of the method (see [21]). We note that the plot for the stability region of the BHT method is given in Figure 1.

3.3. Implementation. We emphasize that the main method and the additional methods specified by (20)-(27) are combined to form block method BHT (29), which is used to solve (1) without requiring starting values and predictors. BHT is implemented in a block-by-block fashion using a Mathematica 10.0 code, enhanced by the feature NSolve[] for linear problems while nonlinear problems were solved by Newton's method enhanced by the feature FindRoot [] (see Keiper and Gear [35]). Mathematica can symbolically

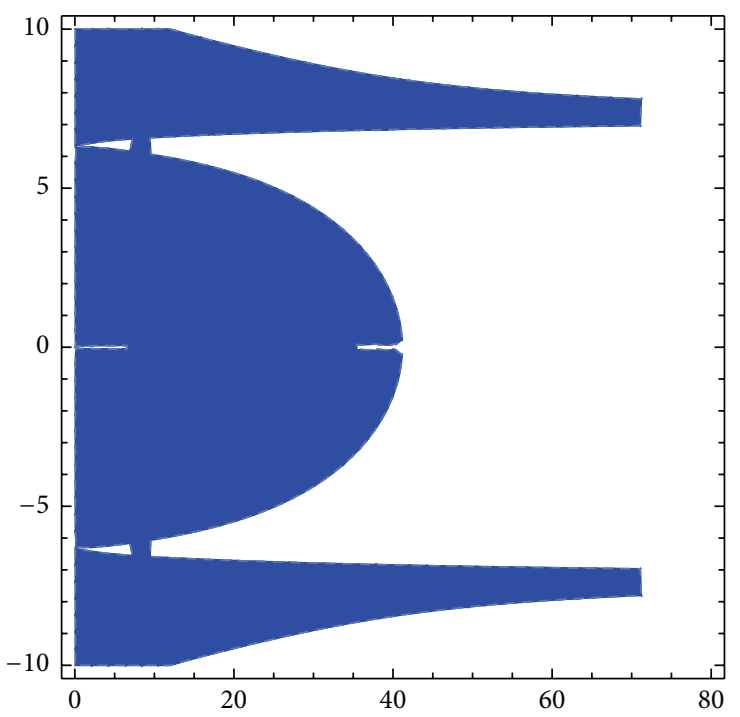

FIGURE 1: The stability region for the BHT plotted in the $(q, u)$-plane.

compute derivatives and so the entries of the Jacobian matrix which involve partial derivatives are automatically generated. In what follows, we summarize how BHT is applied.

Step 1. Choose $N, h=(b-a) / N$, and the number of blocks $\Gamma=N / 2$. Using (29), $n=0, \mu=0$, the values of $\left(y_{1 / 2}, y_{1}, y_{3 / 2}, y_{2}\right)^{T}$ and $\left(y_{1 / 2}^{\prime}, y_{1}^{\prime}, y_{3 / 2}^{\prime}, y_{2}^{\prime}\right)^{T}$ are simultaneously obtained over the subinterval $\left[x_{0}, x_{2}\right]$, as $y_{0}$ and $y_{0}^{\prime}$ are known from IVP (1).

Step 2. For $n=2, \mu=1$, the values of $\left(y_{5 / 2}, y_{3}, y_{7 / 2}, y_{4}\right)^{T}$ and $\left(y_{5 / 2}^{\prime}, y_{3}^{\prime}, y_{7 / 2}^{\prime}, y_{4}^{\prime}\right)^{T}$ are simultaneously obtained over the subinterval $\left[x_{2}, x_{4}\right]$, as $y_{2}$ and $y_{2}^{\prime}$ are known from the previous block.

Step 3. The process is continued for $n=4, \ldots, N-2$ and $\mu=2, \ldots, \Gamma$ to obtain the numerical solution to (1) on the subintervals $\left[x_{0}, x_{2}\right],\left[x_{2}, x_{4}\right], \ldots,\left[x_{N-2}, x_{N}\right]$.

\section{Numerical Examples}

In this section, numerical experiments are performed using a code in Mathematica 10.0 to illustrate the accuracy and efficiency of the method.

Example 1. We consider the following inhomogeneous IVP by Simos [8]:

$$
\begin{aligned}
y^{\prime \prime} & =-100 y+99 \sin (x), \\
y(0) & =1, \\
y^{\prime}(0) & =11,
\end{aligned}
$$




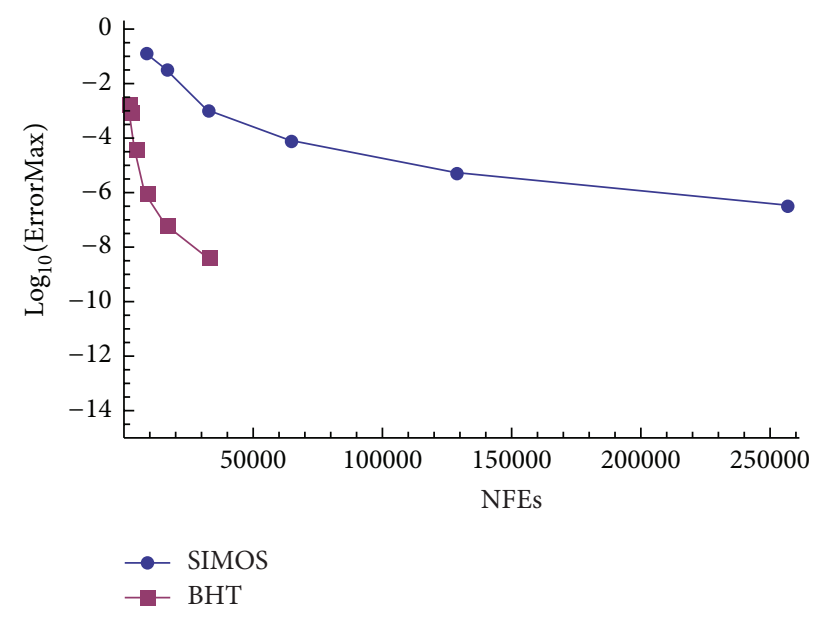

Figure 2: Efficiency curve for Example 1.

TABLE 1: Results, with $\omega=10$, for Example 1.

\begin{tabular}{lccc}
\hline \multicolumn{2}{c}{ Our method } & \multicolumn{2}{c}{ Simos [8] } \\
$N$ & Err & $N$ & Err \\
\hline 1000 & $1.9 \times 10^{-3}$ & 1000 & $1.4 \times 10^{-1}$ \\
2000 & $8.9 \times 10^{-6}$ & 2000 & $3.5 \times 10^{-2}$ \\
4000 & $4.2 \times 10^{-8}$ & 4000 & $1.1 \times 10^{-3}$ \\
8000 & $9.7 \times 10^{-11}$ & 8000 & $8.4 \times 10^{-5}$ \\
16000 & $6.7 \times 10^{-11}$ & 16000 & $5.5 \times 10^{-6}$ \\
32000 & $4.3 \times 10^{-13}$ & 32000 & $3.5 \times 10^{-7}$ \\
\hline
\end{tabular}

where the analytical solution is given by

$$
\text { Exact: } y(x)=\cos (10 x)+\sin (10 x)+\sin (x) \text {. }
$$

This example was solved using the order 5 BHT and the end-point errors $\left(\operatorname{Err}=\left|y\left(x_{N}\right)-y_{N}\right|\right)$ obtained were compared to the order 4 exponentially fitted method given in Simos [8]. In Table 1, it is shown that BHT is more efficient than the method in Simos [8]. We also compare the computational efficiency of the two methods in Figure 2 by considering the FNEs (number of function evaluations) over $N$ integration steps for each method. Our method (BHT) requires fewer number of function evaluations. Hence, for this example, BHT performs better.

Example 2. We consider the nonlinear Duffing equation which was also solved by Simos [8] and Ixaru and Berghe [31]:

$$
\begin{aligned}
y^{\prime \prime}+y+y^{3} & =B \cos (\Omega x), \\
y(0) & =C_{0}, \\
y^{\prime}(0) & =0 .
\end{aligned}
$$

The analytical solution is given by

$$
\text { Exact: } \begin{aligned}
y(x)= & C_{1} \cos (\Omega x)+C_{2} \cos (3 \Omega x) \\
& +C_{3} \cos (5 \Omega x)+C_{4} \cos (7 \Omega x),
\end{aligned}
$$

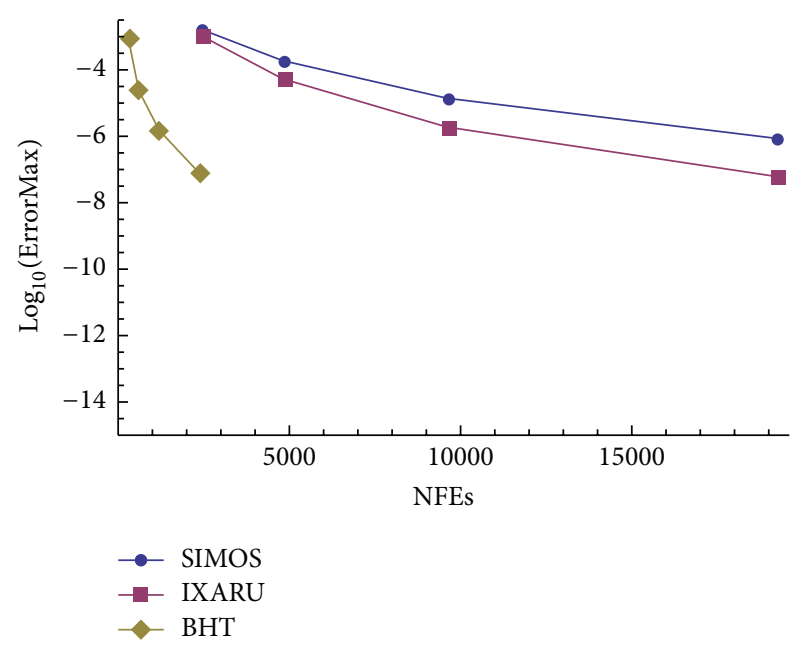

FIgURE 3: Efficiency curves for Example 2.

TABle 2: Results, with $\omega=1.01$, for Example 2.

\begin{tabular}{lccccc}
\hline \multicolumn{2}{c}{ Our method } & \multicolumn{2}{c}{ Simos [8] } & \multicolumn{2}{c}{ Ixaru and Berghe [31] } \\
$N$ & Err & $N$ & Err & $N$ & Err \\
\hline 300 & $7.7 \times 10^{-5}$ & 300 & $1.7 \times 10^{-3}$ & 300 & $1.1 \times 10^{-3}$ \\
600 & $1.7 \times 10^{-6}$ & 600 & $1.9 \times 10^{-4}$ & 600 & $5.4 \times 10^{-5}$ \\
1200 & $1.4 \times 10^{-8}$ & 1200 & $1.4 \times 10^{-5}$ & 1200 & $1.9 \times 10^{-6}$ \\
2400 & $1.9 \times 10^{-10}$ & 2400 & $8.7 \times 10^{-7}$ & 2400 & $6.2 \times 10^{-8}$ \\
\hline
\end{tabular}

where $\Omega=1.01, B=0.002, C_{0}=0.200426728069, C_{1}=$ $0.200179477536, C_{2}=0.246946143 \times 10^{-3}, C_{3}=0.304016 \times$ $10^{-6}$, and $C_{4}=0.374 \times 10^{-9}$. We choose $\omega=1.01$.

We compare the end-point global errors for our method with those of Simos [8] and Ixaru and Berghe [31]. We see from Table 2 that the results produced by our method are competitive to those given in Simos [8] and Ixaru and Berghe [31]. Hence our method is more accurate and efficient as demonstrated in Figure 3.

Example 3. We consider the nonlinear perturbed system on the range $[0,10]$, with $\varepsilon=10^{-3}$ that was also solved in Fang et al. [21]:

$$
\begin{aligned}
y_{1}^{\prime \prime}+25 y_{1}+\varepsilon\left(y_{1}^{2}+y_{2}^{2}\right) & =\varepsilon \varphi_{1}(x), \\
y_{1}(0) & =1, \\
y_{1}^{\prime}(0) & =0, \\
y_{2}^{\prime \prime}+25 y_{2}+\varepsilon\left(y_{1}^{2}+y_{2}^{2}\right) & =\varepsilon \varphi_{2}(x), \\
y_{2}(0) & =\varepsilon, \\
y_{2}^{\prime}(0) & =5,
\end{aligned}
$$


TABLE 3: A comparison of methods for Example 3.

\begin{tabular}{|c|c|c|c|c|c|}
\hline \multicolumn{2}{|c|}{ ARKN5(3) } & \multicolumn{2}{|c|}{ TFARKN5(3) } & \multicolumn{2}{|c|}{ BHT } \\
\hline$N$ (rejected) & $-\log _{10}($ Err $)$ & $N$ (rejected) & $-\log _{10}($ Err $)$ & $N$ & $-\log _{10}($ Err $)$ \\
\hline $42(15)$ & 2.82 & $29(6)$ & 2.78 & 50 & 3.42 \\
\hline $86(7)$ & 4.96 & $88(9)$ & 5.33 & 100 & 4.61 \\
\hline $260(5)$ & 7.16 & $262(8)$ & 7.85 & 260 & 7.52 \\
\hline $812(3)$ & 9.37 & $811(4)$ & 10.38 & 810 & 10.43 \\
\hline
\end{tabular}

where

$$
\begin{aligned}
\varphi_{1}(x)= & 1+\varepsilon^{2}+2 \varepsilon \sin \left(5 x+x^{2}\right)+2 \cos \left(x^{2}\right) \\
& +\left(25-4 x^{2}\right) \sin \left(x^{2}\right), \\
\varphi_{2}(x)= & 1+\varepsilon^{2}+2 \varepsilon \sin \left(5 x+x^{2}\right)-2 \sin \left(x^{2}\right) \\
& +\left(25-4 x^{2}\right) \cos \left(x^{2}\right),
\end{aligned}
$$

and the exact solution is given by $y_{1}(x)=\cos (5 x)+\varepsilon \sin \left(x^{2}\right)$, $y_{2}(x)=\sin (5 x)+\varepsilon \cos \left(x^{2}\right)$, representing a periodic motion of constant frequency with small perturbation of variable frequency.

This problem was solved using the BHT and the maximum global errors $(\operatorname{Err}=\operatorname{Max}|y(x)-y|)$ obtained were compared to the variable step-size trigonometrically fitted Runge-Kutta-Nyström method (TFARKN5(3)) given in Fang et al. [21] and a Runge-Kutta-Nyström method (ARKN5(3)) which was constructed by Franco [15]. In Table 3, the maximum global errors for the three methods are compared. In general, the TFARKN5(3) and ARKN5(3) are expected to perform better because of their variable-step implementation advantage. Nevertheless, the BHT which is implemented in fixed step-size mode is highly competitive to these methods.

Example 4. We consider the following two-body problem which was solved by Ozawa $[25]$ on $[0,50 \pi]$ :

$$
\begin{aligned}
& y_{1}^{\prime \prime}=-\frac{y_{1}}{r^{3}}, \\
& y_{2}^{\prime \prime}=-\frac{y_{2}}{r^{3}},
\end{aligned}
$$$$
r=\sqrt{y_{1}^{2}+y_{2}^{2}}
$$

$$
\begin{aligned}
& y_{1}(0)=1-e, \\
& y_{1}^{\prime}(0)=0, \\
& y_{2}(0)=0, \\
& y_{2}^{\prime}(0)=\sqrt{\frac{1+e}{1-e}},
\end{aligned}
$$

TABLE 4: Steps and absolute errors, with $\omega=1$, for Example $4[0,50 \pi]$.

\begin{tabular}{lccccc}
\hline \multicolumn{2}{c}{ Our method } & \multicolumn{2}{c}{ FESDIRK4(3) [25] } & \multicolumn{2}{c}{ ESDIRK4(3) [25] } \\
Steps & Err & Steps & Err & Steps & Err \\
\hline 220 & $3.52 \times 10^{-5}$ & 170 & $2.866 \times 10^{-1}$ & 277 & $2.153 \times 10^{0}$ \\
300 & $1.13 \times 10^{-6}$ & 225 & $7.846 \times 10^{-3}$ & 496 & $1.494 \times 10^{-1}$ \\
400 & $1.03 \times 10^{-7}$ & 381 & $1.399 \times 10^{-3}$ & 884 & $9.359 \times 10^{-3}$ \\
600 & $3.49 \times 10^{-10}$ & 680 & $1.690 \times 10^{-4}$ & 1573 & $6.200 \times 10^{-4}$ \\
800 & $1.14 \times 10^{-11}$ & 1207 & $1.846 \times 10^{-5}$ & 2796 & $4.416 \times 10^{-5}$ \\
1000 & $7.68 \times 10^{-13}$ & 2144 & $1.938 \times 10^{-6}$ & 4970 & $3.412 \times 10^{-6}$ \\
1200 & $2.8 \times 10^{-14}$ & 3806 & $1.993 \times 10^{-7}$ & 8833 & $2.848 \times 10^{-7}$ \\
2400 & $1.02 \times 10^{-13}$ & 6762 & $2.021 \times 10^{-8}$ & 15706 & $2.530 \times 10^{-8}$ \\
\hline
\end{tabular}
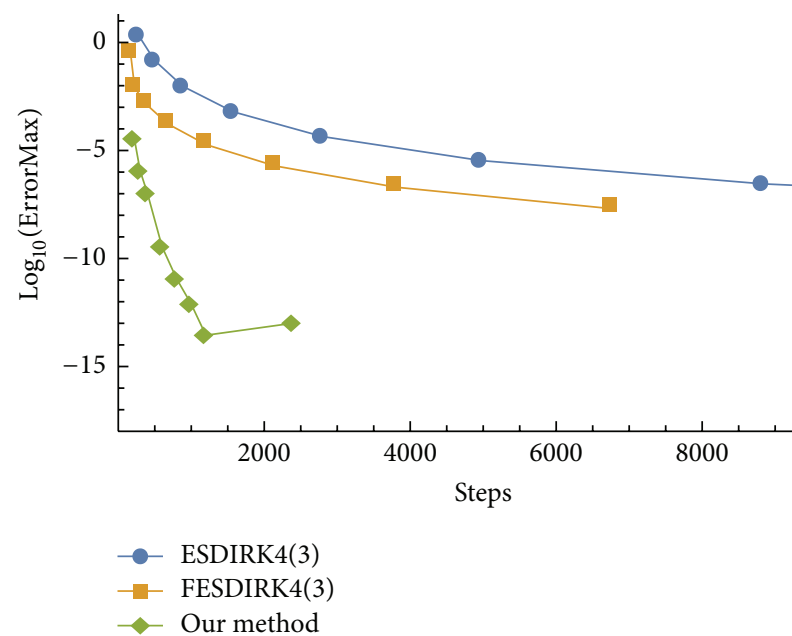

FIGURE 4: Efficiency curve for Example 4.

where $e(0 \leq e<1)$ is an eccentricity. The exact solution of this problem is

$$
\text { Exact: } \begin{aligned}
y_{1}(x) & =\cos (k)-e, \\
y_{2}(x) & =\sqrt{1-e^{2}} \sin (k),
\end{aligned}
$$

where $k$ is the solution of Kepler's equation $k=x+e \sin (k)$. We choose $\omega=1$.

We show in Table 4 that the results obtained using the BHT method are more accurate than the explicit singly diagonally implicit Runge-Kutta (ESDIRK) and the functionally fitted ESDIRK (FESDIRK) methods given in Ozawa [25]. In Figure 4, we also illustrate the efficiency advantage of the BHT method over those in Ozawa [25]. 
TABLE 5: Results, with $\omega=1$, for Example 5.

\begin{tabular}{lccc}
\hline \multicolumn{2}{c}{ Our method } & \multicolumn{2}{c}{ Vigo-Aguiar and Ramos [32] } \\
$N$ & Err & $N$ & Err \\
\hline 67 & $1.14 \times 10^{-9}$ & 67 & $7.11 \times 10^{-7}$ \\
82 & $3.5 \times 10^{-10}$ & 82 & $9.26 \times 10^{-8}$ \\
97 & $1.3 \times 10^{-10}$ & 97 & $87.8 \times 10^{-9}$ \\
112 & $5.5 \times 10^{-11}$ & 112 & $1.12 \times 10^{-10}$ \\
125 & $2.9 \times 10^{-11}$ & 125 & $2.71 \times 10^{-11}$ \\
\hline
\end{tabular}

\subsection{Problems Where $y^{\prime}$ Appears Explicitly}

Example 5 (Bessel's IVP). We consider the Bessel differential equation that was also solved by Vigo-Aguiar and Ramos [11]:

$$
\begin{aligned}
& x^{2} y^{\prime \prime}+x y^{\prime}+\left(x^{2}-0.25\right) y=0, \\
& y(1)=\sqrt{\frac{2}{\pi}} \sin 1 \approx 0.671397071418031, \\
& y^{\prime}(1)=\frac{(2 \cos 1-\sin 1)}{2 \pi} \approx 0.0954005144474746, \\
& x \in[1,8],
\end{aligned}
$$

where the exact (analytical) solution is given by

$$
\text { Exact: } y(x)=J_{1 / 2}(x)=\sqrt{\frac{2}{\pi x}} \sin (x) \text {. }
$$

This problem was chosen to demonstrate the performance of our method on the general second-order IVP with variable coefficients. We compare our results with the variable-step Falkner method of order eight that was implemented in predictor-corrector mode by Vigo-Aguiar and Ramos [32]. The results displayed in Table 5 show that the BHT method performs better.

Example 6. We consider the harmonic oscillator with frequency $\Omega$ and small perturbation $\delta$ that was solved in Franco [15] and Guo and Yan [36]:

$$
\begin{aligned}
& y^{\prime \prime}+\delta y^{\prime}+\Omega^{2} y=0, \\
& y(0)=0, \\
& y^{\prime}(0)=-\frac{\delta}{2}, \\
& x \in[0,1000],
\end{aligned}
$$

where the analytical solution is given by

$$
\text { Exact: } y(x)=e^{(\delta / 2) x} \cos \left(\Omega^{2}-\frac{\delta^{2}}{4}\right),
$$

where $\Omega=1, \delta=10^{-6}$, and $\delta=10^{-10}$. The problem was solved in Guo and Yan [36] using ARKN method. In Table 6, the errors are compared at $x=1000$. We observed that the BHT is competitive with the order 5 Runge-Kutta-Nyström method.

\subsection{Hyperbolic PDEs}

Example 7. We consider the given Telegraph equation (see Ding et al. [33]):

$$
\begin{aligned}
\frac{\partial^{2} u}{\partial t^{2}} & +2 \pi \frac{\partial u}{\partial t}+\pi^{2} u \\
& =\frac{\partial^{2} u}{\partial x^{2}}+\pi^{2} \sin (\pi x)(\sin (\pi t)+\cos (\pi t))
\end{aligned}
$$

$$
0 \leq x \leq 1,0 \leq t \leq 1
$$

The exact solution is given by $u(x, y)=\sin (\pi x) \sin (\pi t)$.

In order to solve this PDE using the BHT, we carry out the semidiscretization of the spatial variable $x$ using the secondorder finite difference method to obtain the following secondorder system in the second variable $t$ :

$$
\begin{aligned}
& \frac{\partial^{2} u_{m}}{\partial t^{2}}+2 \pi \frac{\partial u_{m}}{\partial t}+\pi^{2} u_{m}-\frac{\left(u_{m+1}-2 u_{m}+u_{m-1}\right)}{(\Delta x)^{2}} \\
& \quad=g_{m}, \quad 0<t<1, m=1, \ldots, M-1, \\
& u\left(x_{m}, 0\right)=u_{m}, \\
& u_{t}\left(x_{m}, 0\right)=u_{m}^{\prime},
\end{aligned}
$$

where $\Delta x=(b-a) / M, x_{m}=a+m \Delta x, m=0,1, \ldots, M, \mathbf{u}=$ $\left[u_{1}(t), \ldots, u_{M}(t)\right]^{T}, \mathbf{g}=\left[g_{1}(t), \ldots, g_{m}(t)\right]^{T}, u_{m}(t) \approx u\left(x_{m}, t\right)$, and $g_{m}(t) \approx g\left(x_{m}, t\right)=\pi^{2} \sin \left(\pi x_{m}\right)(\sin (\pi t)+\cos (\pi t))$, which can be written in the form

$$
\mathbf{u}^{\prime \prime}=\mathbf{f}\left(t, \mathbf{u}, \mathbf{u}^{\prime}\right)
$$

subject to the boundary conditions $\mathbf{u}\left(t_{0}\right)=\mathbf{u}_{0}, \mathbf{u}^{\prime}\left(t_{0}\right)=\mathbf{u}_{0}^{\prime}$, where $\mathbf{f}\left(t, \mathbf{u}, u^{\prime}\right)=\mathbf{A} \mathbf{u}+\mathbf{g}$, and $\mathbf{A}$ is $M-1 \times M-1$, matrix arising from the semidiscretized system, and $\mathbf{g}$ is a vector of constants.

The boundary conditions are chosen accordingly. This example was chosen to demonstrate that the BHT can be used to solve the Telegraph equation. In Table 7 , the results produced by the BHT using $\Delta t=1 / 100$ and space step $\Delta x=$ $1 / 100$ are compared to scheme (3.12) $\left(\lambda_{1}=1 / 12\right.$, and $\lambda_{2}=$ $5 / 6)$, time step $\Delta t=1 / 200$, and space step $\Delta x=1 / 100$, given in Ding et al. [33]. It is obvious from Table 5 that the BHT is more accurate than the method given in [33]. Moreover, the errors produced by BHT method using $\Delta t=1 / 100$ and space step $\Delta x=1 / 100$ are given in Figure 5. 
TABLE 6: Errors at $x=1000$, for Example 6.

\begin{tabular}{lccccc}
\hline & \multicolumn{1}{c}{ BHT } & & \multicolumn{2}{c}{ ARKN } \\
$h$ & Error $\left(\delta=10^{-6}\right)$ & Error $\left(\delta=10^{-10}\right)$ & $h$ & Error $\left(\delta=10^{-6}\right)$ & Error $\left(\delta=10^{-10}\right)$ \\
\hline 1 & $4.12 \times 10^{-8}$ & $1.11 \times 10^{-11}$ & $1 / 2$ & $9.05 \times 10^{-8}$ & $9.00 \times 10^{-12}$ \\
$1 / 2$ & $7.06 \times 10^{-10}$ & $2.19 \times 10^{-13}$ & $1 / 4$ & $5.43 \times 10^{-9}$ & $7.06 \times 10^{-13}$ \\
$1 / 4$ & $1.23 \times 10^{-11}$ & $3.12 \times 10^{-13}$ & $1 / 8$ & $2.03 \times 10^{-10}$ & $2.87 \times 10^{-13}$ \\
$1 / 8$ & $5.23 \times 10^{-12}$ & $5.44 \times 10^{-12}$ & $1 / 16$ & $7.25 \times 10^{-12}$ & $3.56 \times 10^{-13}$ \\
$1 / 16$ & $5.62 \times 10^{-12}$ & $2.94 \times 10^{-12}$ & $1 / 32$ & $3.45 \times 10^{-13}$ & $5.91 \times 10^{-13}$ \\
\hline
\end{tabular}

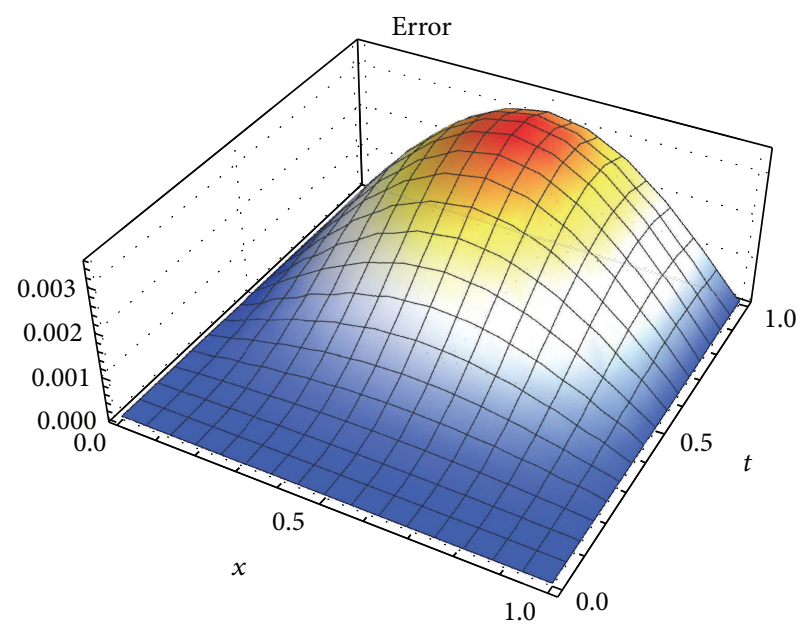

(a) $N=M=10$

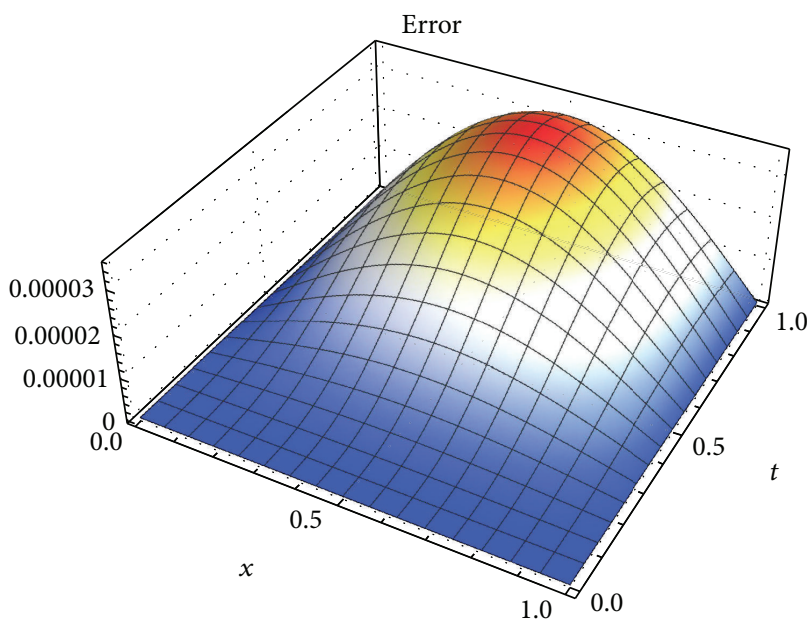

(b) $N=M=100$

Figure 5: Absolute errors for Example 7.

TABLE 7: Results, with $\omega=\pi$, for Example 7 .

\begin{tabular}{lcc}
\hline & Our method & Ding et al. [33] \\
$x$ & Err & Err \\
\hline 0.2 & $2.06 \times 10^{-10}$ & $9.62 \times 10^{-10}$ \\
0.4 & $3.33 \times 10^{-10}$ & $1.56 \times 10^{-9}$ \\
0.6 & $3.33 \times 10^{-10}$ & $1.56 \times 10^{-9}$ \\
0.8 & $2.06 \times 10^{-10}$ & $9.62 \times 10^{-10}$ \\
\hline
\end{tabular}

Example 8. We consider the wave equation given in Franco [15]. A problem representing a vibrating string with speed $\omega$ is given by

$$
\begin{aligned}
\frac{\partial^{2} u}{\partial t^{2}}-x(1-x) \frac{\partial^{2} u}{\partial x^{2}}+\left(\omega^{2}-2\right) u & =0 \\
0 & <x<1,0<t \leq 5, \\
u(0, t) & =0, \\
u(1, t) & =0, \\
u(x, 0) & =x(1-x), \\
u_{t}(x, 0) & =0,
\end{aligned}
$$

where the initial and Dirichlet boundary conditions have been chosen such that the solution is given by $u(x, t)=x(1-$ $x) \cos \omega t$. In order to solve this PDE using the BHT, we carry out the semidiscretization of the spatial variable $x$ using the second-order finite difference method to obtain the following second-order system in the second variable $t$ :

$$
\begin{aligned}
& \frac{\partial^{2} u_{m}}{\partial t^{2}}-x_{m}\left(1-x_{m}\right)+\frac{\left(u_{m+1}-2 u_{m}+u_{m-1}\right)}{(\Delta x)^{2}} \\
& \quad+\left(\omega^{2}-2\right) u_{m}=g_{m}, \quad m=1, \ldots, M-1, \\
& u\left(x_{m}, 0\right)=x_{m}\left(1-x_{m}\right), \\
& u_{t}\left(x_{m}, 0\right)=0, \quad 0<t \leq 5,
\end{aligned}
$$

where $\Delta x=(b-a) / M, x_{m}=a+m \Delta x, m=0,1, \ldots, M, \mathbf{u}=$ $\left[u_{1}(t), \ldots, u_{M}(t)\right]^{T}, \mathbf{g}=\left[g_{1}(t), \ldots, g_{m}(t)\right]^{T}, u_{m}(t) \approx u\left(x_{m}, t\right)$, and $g_{m}(t) \approx g\left(x_{m}, t\right)=0$, which can be written in the form

$$
\mathbf{u}^{\prime \prime}=\mathbf{f}(t, \mathbf{u})
$$

subject to the boundary conditions $\mathbf{u}\left(t_{0}\right)=\mathbf{u}_{0}, \mathbf{u}^{\prime}\left(t_{0}\right)=\mathbf{u}_{0}^{\prime}$, where $\mathbf{f}(t, \mathbf{u})=\mathbf{A} \mathbf{u}+\mathbf{g}$, and $\mathbf{A}$ is $M-1 \times M-1$, matrix arising from the semidiscretized system, and $\mathbf{g}$ is a vector of constants.

In Figure 6, we give the errors produced by the BHT which show that the method performs very well on this problem. 


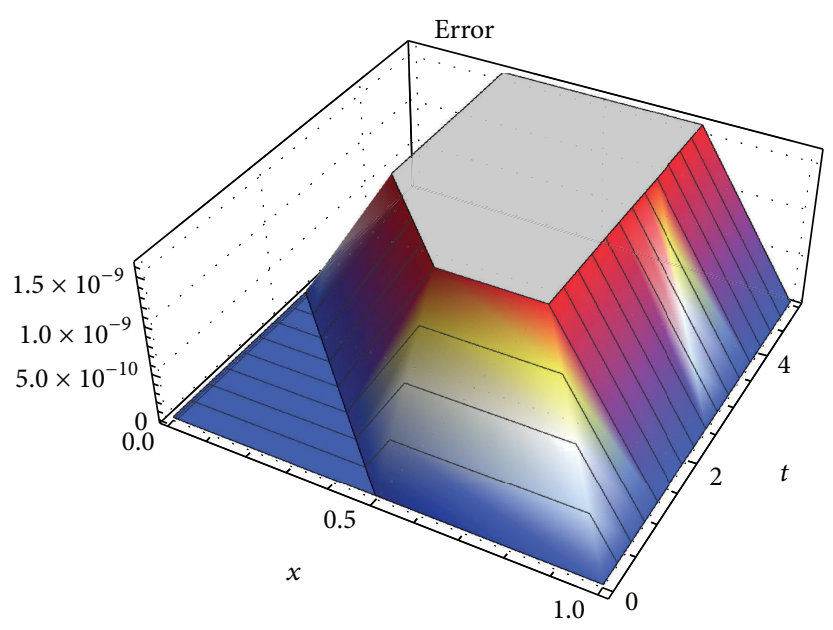

(a) $N=M=2$

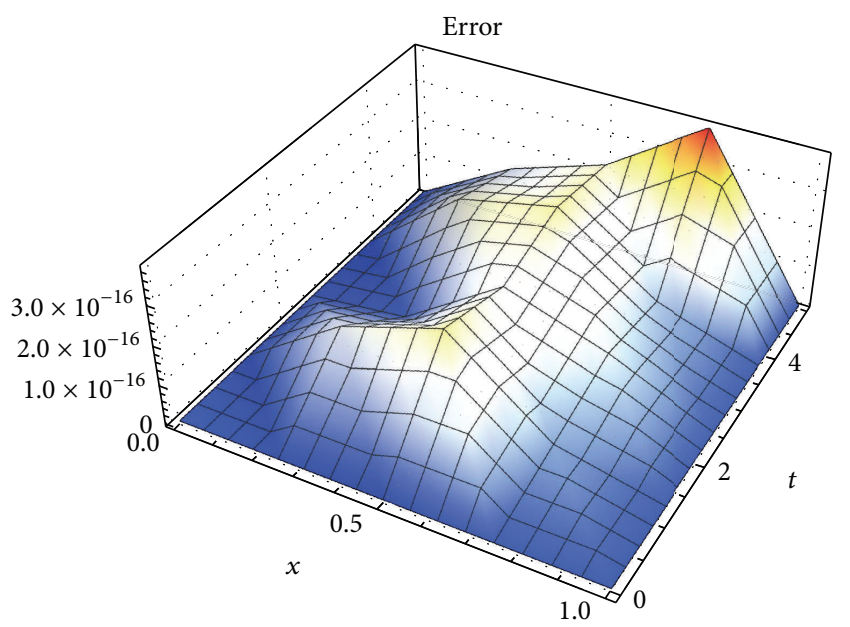

(b) $N=M=4$

Figure 6: Absolute errors for Example 8.

TABLE 8: Comparison of methods for Example 9.

\begin{tabular}{lcccc}
\hline$N$ & Method in [34] & Our method & $N$ & RK4 \\
\hline 10 & $1.12 \times 10^{-4}$ & $2.23 \times 10^{-4}$ & 250 & Overflow \\
20 & $1.69 \times 10^{-6}$ & $3.36 \times 10^{-6}$ & 500 & Overflow \\
40 & $1.22 \times 10^{-8}$ & $2.44 \times 10^{-8}$ & 1000 & Overflow \\
80 & $9.79 \times 10^{-11}$ & $1.96 \times 10^{-10}$ & 2000 & Overflow \\
160 & $1.06 \times 10^{-12}$ & $2.13 \times 10^{-12}$ & 4000 & $1.20 \times 10^{-13}$ \\
320 & $1.54 \times 10^{-14}$ & $2.48 \times 10^{-14}$ & 8000 & $7.94 \times 10^{-15}$ \\
\hline
\end{tabular}

Example 9. We consider the following mildly stiff IVP which was also solved in [34]:

$$
\begin{aligned}
y^{\prime \prime} & =-1001 y^{\prime}-1000 y, \\
y(0) & =1, \\
y^{\prime}(0) & =-1,
\end{aligned}
$$

$$
x \in[0,10]
$$

Exact: $y(x)=e^{-x}$.

This example is given to show that the method still performs well on problems with nontrigonometric solutions. The problem was solved using the BHT and the results obtained were compared with the polynomial based method given in [34] and the standard fourth-order Runge-Kutta method (RK4). The results given in Table 8 show that the BHT is competitive with the method in [34] and is superior to RK4 which are designed for problems with nontrigonometric solutions.

\section{Conclusion}

We have presented a BHT method whose coefficients are functions of the frequency and the step-size for directly solving general second-order initial value problems (IVPs), including systems arising from the semidiscretization of hyperbolic PDEs, such as the Telegraph equation. The BHT is implemented in a block-by-block fashion; in this way, the method does not suffer from the disadvantages of requiring starting values and predictors which are inherent in predictor-corrector methods. We have also shown that the BHT method has a reasonably wide stability region and enjoys accuracy and efficiency advantages when compared to existing methods in the literature. Our future research will be to incorporate a technique for accurately estimating the frequency as suggested in $[37,38]$ as well as implementing the method in a variable-step mode.

\section{Conflict of Interests}

The authors declare that there is no conflict of interests regarding the publication of this paper.

\section{References}

[1] J. D. Lambert, Numerical Methods for Ordinary Differential Systems, John Wiley \& Sons, New York, NY, USA, 1991.

[2] J. D. Lambert, Computational Methods in Ordinary Differential Equations, John Wiley \& Sons, New York, NY, USA, 1973.

[3] E. Hairer and G. Wanner, Solving Ordinary Differential Equations II, vol. 14 of Springer Series in Computational Mathematics, Springer, 2nd edition, 1996.

[4] E. Hairer, "A one-step method of order 10 for $y^{\prime \prime}=f(x, y)$," IMA Journal of Numerical Analysis, vol. 2, pp. 83-94, 1982.

[5] L. Brugnano and D. Trigiante, Solving ODEs by Multistep Initial and Boundary Value Methods, Gordon \& Breach, Amsterdam, The Netherlands, 1998.

[6] L. Brugnano and D. Trigiante, "Block implicit methods for ODEs," in Recent Trends in Numerical Analysis, D. Trigiante, Ed., pp. 81-105, Nova Science Publishers, New York, NY, USA, 2001.

[7] E. Hairer, S. P. Norsett, and G. Wanner, Solving Ordinary Differential Equations I, Nonstiff Problems, Springer, Berlin, Germany, 1993. 
[8] T. E. Simos, "An exponentially-fitted Runge-Kutta method for the numerical integration of initial-value problems with periodic or oscillating solutions," Computer Physics Communications, vol. 115, no. 1, pp. 1-8, 1998.

[9] J. D. Lambert and A. Watson, "Symmetric multistip methods for periodic initial value problems," IMA Journal of Applied Mathematics, vol. 18, no. 2, pp. 189-202, 1976.

[10] E. H. Twizell and A. Q. M. Khaliq, "Multiderivative methods for periodic initial value problems," SIAM Journal on Numerical Analysis, vol. 21, no. 1, pp. 111-122, 1984.

[11] J. Vigo-Aguiar and H. Ramos, "Dissipative Chebyshev exponential-fitted methods for numerical solution of second-order differential equations," Journal of Computational and Applied Mathematics, vol. 158, no. 1, pp. 187-211, 2003.

[12] D. O. Awoyemi, "A new sixth-order algorithm for general second order ordinary differential equations," International Journal of Computer Mathematics, vol. 77, no. 1, pp. 117-124, 2001.

[13] M. M. Chawla and S. R. Sharma, "Families of three-stage third order Runge-Kutta-Nystrom methods for $y n=f\left(x, y, y^{\prime}\right)$," Journal of the Australian Mathematical Society, vol. 26, pp. 375386, 1985.

[14] S. M. Mahmoud and M. S. Osman, "On a class of splinecollocation methods for solving second-order initial-value problems," International Journal of Computer Mathematics, vol. 86, no. 4, pp. 616-630, 2009.

[15] J. M. Franco, "Runge-Kutta-Nyström methods adapted to the numerical integration of perturbed oscillators," Computer Physics Communications, vol. 147, no. 3, pp. 770-787, 2002.

[16] S. N. Jator, "A continuous two-step method of order 8 with a block extension for $y^{\prime \prime}=f\left(x, y, y^{\prime}\right)$," Applied Mathematics and Computation, vol. 219, pp. 781-791, 2012.

[17] J. P. Coleman and S. C. Duxbury, "Mixed collocation methods for $y^{\prime \prime}=f(x, y)$," Journal of Computational and Applied Mathematics, vol. 126, pp. 47-75, 2000.

[18] J. P. Coleman and L. G. Ixaru, "P-stability and exponentialfitting methods for $y n=f(x, y)$," IMA Journal of Numerical Analysis, vol. 16, pp. 179-199, 1996.

[19] T. E. Simos, "Dissipative trigonometrically-fitted methods for second order IVPs with oscillating solution," International Journal of Modern Physics C, vol. 13, no. 10, pp. 1333-1345, 2002.

[20] G. Vanden Berghe, L. G. Ixaru, and M. van Daele, "Optimal implicit exponentially-fitted Runge-Kutta methods," Computer Physics Communications, vol. 140, no. 3, pp. 346-357, 2001.

[21] Y. Fang, Y. Song, and X. Wu, "A robust trigonometrically fitted embedded pair for perturbed oscillators," Journal of Computational and Applied Mathematics, vol. 225, no. 2, pp. 347-355, 2009.

[22] H. S. Nguyen, R. B. Sidje, and N. H. Cong, "Analysis of trigonometric implicit Runge-Kutta methods," Journal of Computational and Applied Mathematics, vol. 198, no. 1, pp. 187-207, 2007.

[23] H. Ramos and J. Vigo-Aguiar, "A trigonometrically-fitted method with two frequencies, one for the solution and another one for the derivative," Computer Physics Communications, vol. 185, no. 4, pp. 1230-1236, 2014.

[24] J. M. Franco and I. Gómez, "Trigonometrically fitted nonlinear two-step methods for solving second order oscillatory IVPs," Applied Mathematics and Computation, vol. 232, pp. 643-657, 2014.
[25] K. Ozawa, "A functionally fitted three-stage explicit singly diagonally implicit Runge-Kutta method," Japan Journal of Industrial and Applied Mathematics, vol. 22, no. 3, pp. 403-427, 2005.

[26] S. N. Jator, S. Swindle, and R. French, "Trigonometrically fitted block Numerov type method for $y^{\prime \prime}=f\left(x, y, y^{\prime}\right)$," Numerical Algorithms, vol. 62, no. 1, pp. 13-26, 2013.

[27] S. N. Jator, "Implicit third derivative Runge-Kutta-Nyström method with trigonometric coefficients," Numerical Algorithms, vol. 70, no. 1, pp. 133-150, 2015.

[28] F. F. Ngwane and S. N. Jator, "Block hybrid method using trigonometric basis for initial value problems with oscillating solutions," Numerical Algorithms, vol. 63, no. 4, pp. 713-725, 2013.

[29] S. N. Jator, "Block third derivative method based on trigonometric polynomials for periodic initial-value problems," Afrika Matematika, pp. 1-13, 2015.

[30] H. Ramos, Z. Kalogiratou, Th. Monovasilis, and T. E. Simos, "A trigonometrically fitted optimized two-step hybrid block method for solving initialvalue problems of the form $y^{\prime \prime}=$ $f\left(x, y, y^{\prime}\right)$ with oscillatory solutions," AIP Conference Proceedings, vol. 1648, Article ID 810007, 2015.

[31] L. Ixaru and G. V. Berghe, Exponential Fitting, Kluwer Academic Publishers, Dordrecht, The Netherlands, 2004.

[32] J. Vigo-Aguiar and H. Ramos, "Variable stepsize implementation of multistep methods for $y^{\prime \prime}=f\left(x, y, y^{\prime}\right)$," Journal of Computational and Applied Mathematics, vol. 192, no. 1, pp. 114131, 2006.

[33] H.-F. Ding, Y.-X. Zhang, J.-X. Cao, and J.-H. Tian, "A class of difference scheme for solving telegraph equation by new non-polynomial spline methods," Applied Mathematics and Computation, vol. 218, no. 9, pp. 4671-4683, 2012.

[34] S. N. Jator, "On a class of hybrid methods for $y^{\prime \prime}=f\left(x, y, y^{\prime}\right)$," International Journal of Pure and Applied Mathematics, vol. 59, no. 4, pp. 381-395, 2010.

[35] J. B. Keiper and C. W. Gear, "The analysis of generalized backwards difference formula methods applied to Hessenberg form differential-algebraic equations," SIAM Journal on Numerical Analysis, vol. 28, no. 3, pp. 833-858, 1991.

[36] B.-Y. Guo and J.-P. Yan, "Legendre-Gauss collocation method for initial value problems of second order ordinary differential equations," Applied Numerical Mathematics, vol. 59, no. 6, pp. 1386-1408, 2009.

[37] G. V. Berghe, L. G. Ixaru, and H. De Meyer, "Frequency determination and step-length control for exponentially-fitted Runge-Kutta methods," Journal of Computational and Applied Mathematics, vol. 132, no. 1, pp. 95-105, 2001.

[38] H. Ramos and J. Vigo-Aguiar, "On the frequency choice in trigonometrically fitted methods," Applied Mathematics Letters, vol. 23, no. 11, pp. 1378-1381, 2010. 


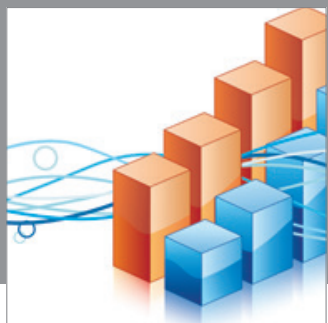

Advances in

Operations Research

mansans

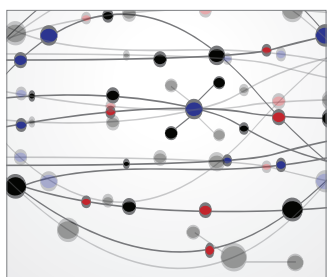

The Scientific World Journal
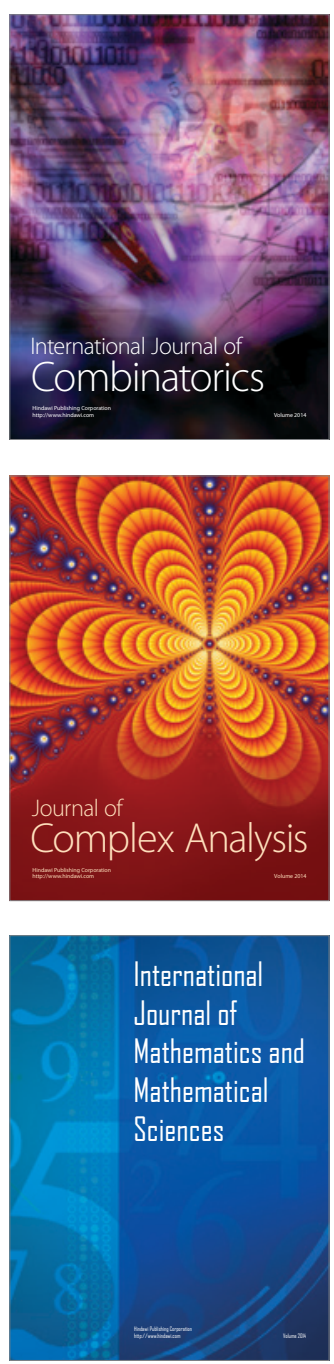
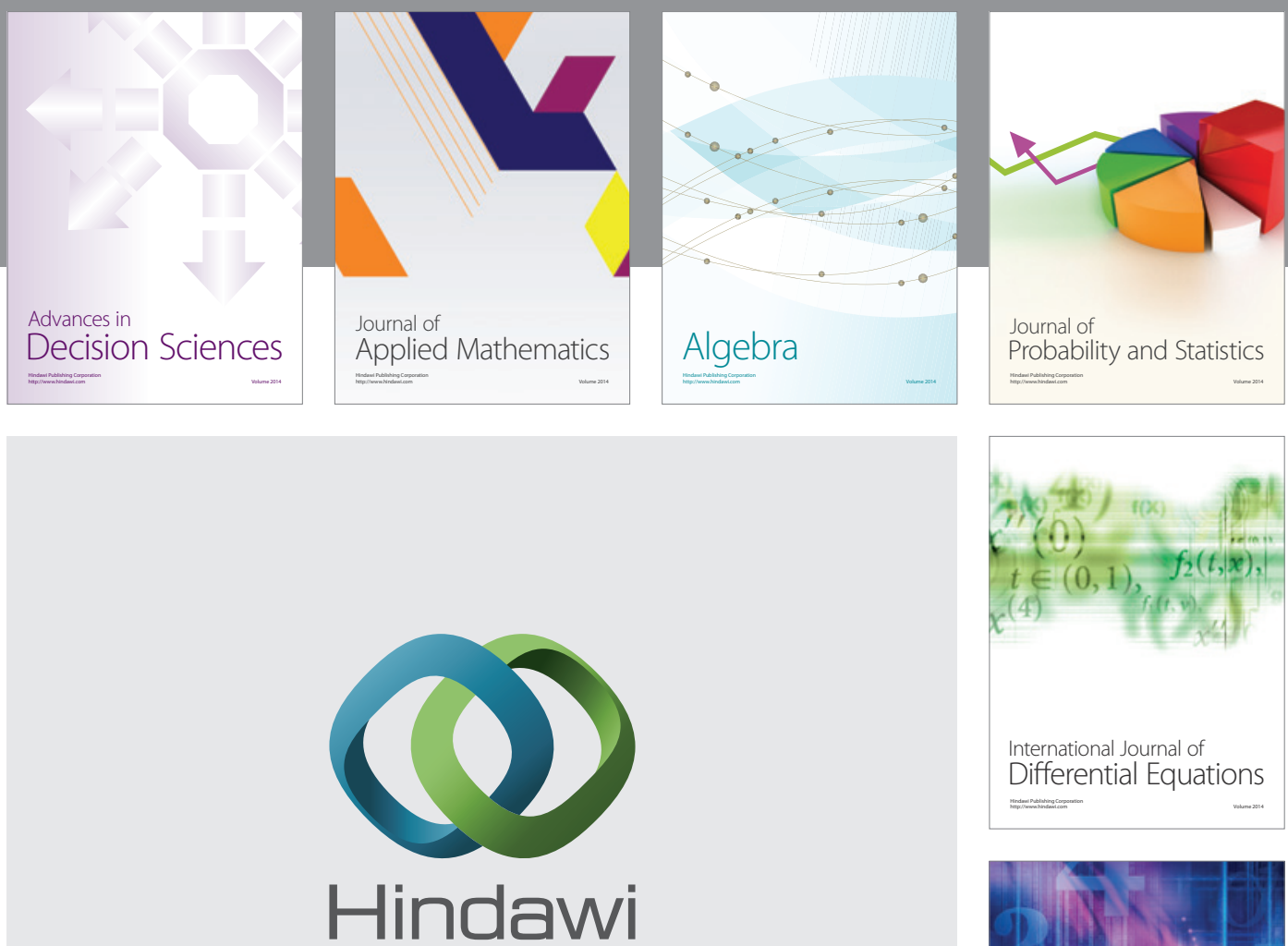

Submit your manuscripts at http://www.hindawi.com
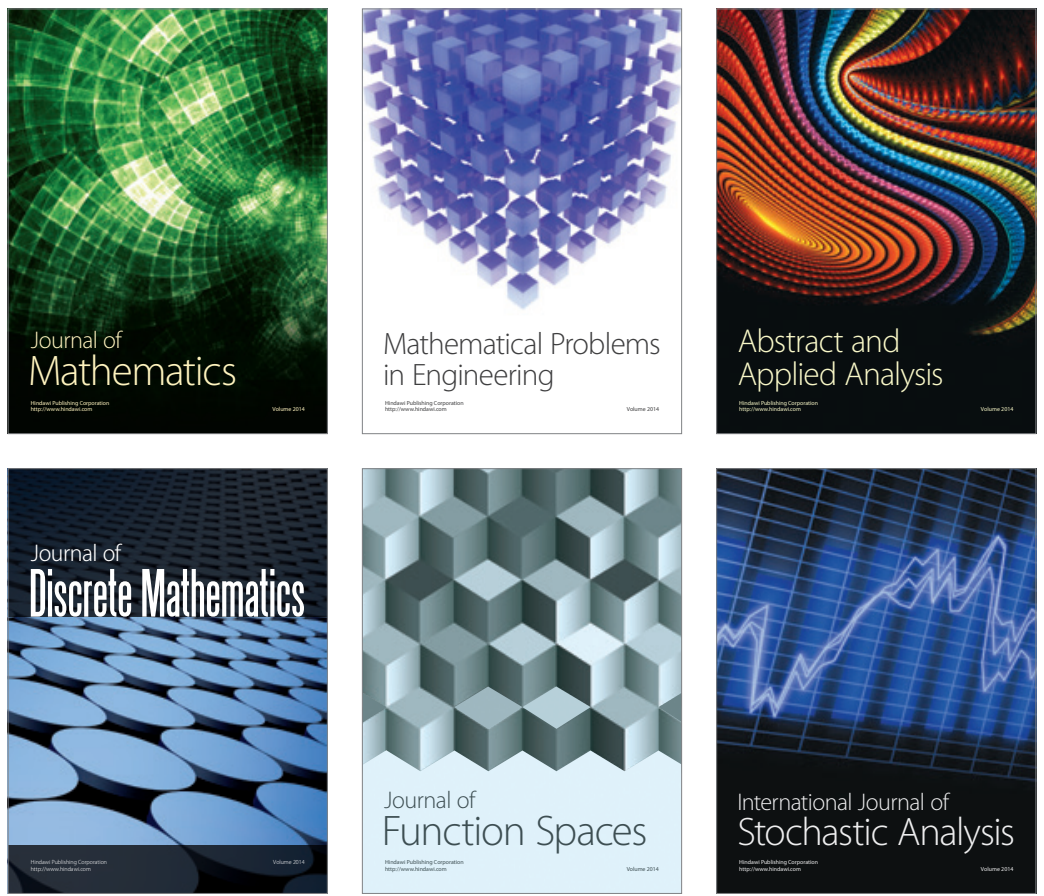

Journal of

Function Spaces

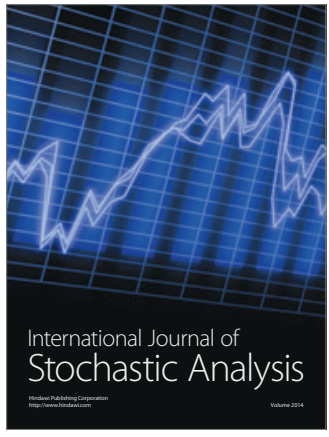

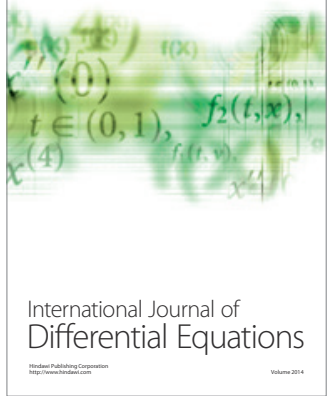
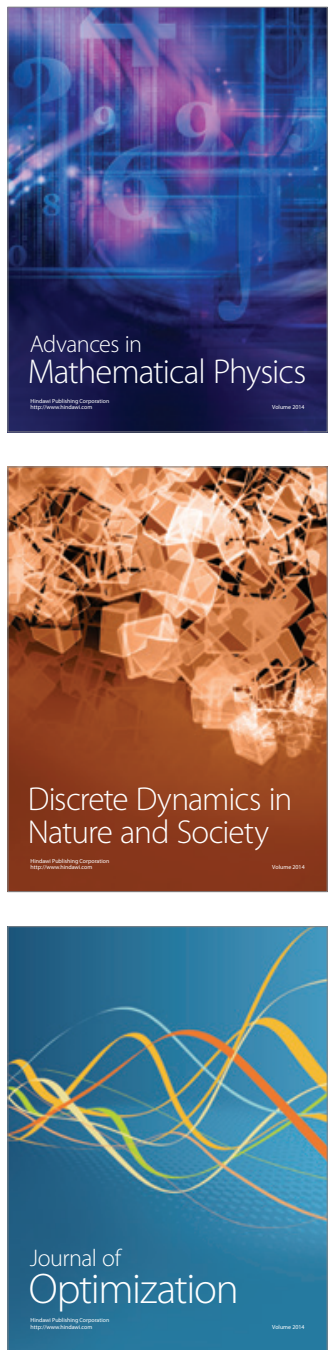